\title{
Generalization of Linear Rosenstark Method of Feedback Amplifier Analysis to Nonlinear One
}

\author{
Andrzej Borys and Zbigniew Zakrzewski
}

\begin{abstract}
This paper deals with an extension of the Rosenstark's linear model of an amplifier to a nonlinear one for the purpose of performing nonlinear distortion analysis. Contrary to an approach using phasors, our method uses the Volterra series. Relying upon the linear model mentioned above, we define first a set of the so-called amplifier's constitutive equations in an operator form. Then, we expand operators using the Volterra series truncated to the first three components. This leads to getting two representations in the time domain, called in-network and inputoutput type descriptions of an amplifier. Afterwards, both of these representations are transferred into the multi-frequency domains. Their usefulness in calculations of any nonlinear distortion measure as, for example, harmonic, intermodulation, and/or cross-modulation distortion is demonstrated. Moreover, we show that they allow a simple calculation of the so-called nonlinear transfer functions in any topology as, for example, of cascade and feedback structures and their combinations occurring in single-, two-, and three-stage amplifiers. Examples of such calculations are given. Finally in this paper, we comment on usage of such notions as nonlinear signals, intermodulation nonlinearity, and on identification of transfer function poles and zeros lying on the frequency axis with related real-valued frequencies.
\end{abstract}

Keywords-weakly nonlinear amplifiers, nonlinear Rosenstark model, nonlinear distortion analysis, harmonic distortion, constitutive equations, Volterra series

\section{INTRODUCTION}

$\mathbf{I}$ $\mathrm{N}$ his well known paper [1] and a book [2], Rosenstark developed an unconventional means of analysis of linear feedback amplifiers in the frequency domain. This approach became recently very popular among designers of amplifiers implemented in CMOS and related technologies, see for example [3], [4], [5]. The original Rosenstark's amplifier model is a linear one. So, in such a form, it cannot be used in evaluation of nonlinear distortion occurring in weakly (mildly) nonlinear amplifiers - that is in amplifiers which are not strictly linear ones. Nevertheless, it has been used in [5] as a heuristic tool for developing a specific nonlinear model for calculation of harmonic distortions. Correctness of the so-called nonlinear coefficients of the first-, second-, and third-order calculated with its help has been checked in [5] by confronting them with their counterparts evaluated in the analysis using phasors.

In this paper, we extend the original linear Rosenstark's model to a fully nonlinear one, presenting its general form using operators working on continuous time signals. This

A. Borys and Z. Zakrzewski are with the Faculty of Telecommunications, Computer Science, and Electrical Engineering, University of Technology and Life Sciences (UTP), 85-789 Bydgoszcz, Poland; (e-mails: \{andrzej.borys; zbigniew.zakrzewski\}@utp.edu.pl). operator model exploits four linear operators and two nonlinear ones. Further, the nonlinear operators are assumed to have expansions of a polynomial type, which are afterwards truncated to the first three components. All the operators mentioned above are used in a set of three equations. This set constitutes the so-called weakly nonlinear amplifier description - according to the ideas of Rosenstark [1], [2]. It builds a model called weakly nonlinear one because it involves only nonlinearities of the polynomial type which are of the highest order of three. Further, in this form, the model formulation is in the time domain.

After Chua [6], the aforementioned set is a set of constitutive relations (equations) describing a weakly nonlinear amplifier as a two-port (shortly 2-port). More precisely, it is a simplified set of constitutive equations because it involves only two port variables from the whole number of four occurring in this case. Moreover, it is such a set of relations that contains also (two) internal variables, besides the port ones mentioned above. Further, consistently with the above simplification, none of the six aforementioned operators describes any 2-port circuit element of which the amplifier model is built. Each of them describes a two-terminal (shortly 2-terminal) circuit element independently of weather it is in fact such an element (as for example a nonlinear amplifier conductance) or not (as for instance a nonlinear voltage controlled current source). For those amplifier model components that are 2-ports, these descriptions form equivalent 2 -terminal circuit element representations. We can view them as 2-port-like-2-terminal or block-like-2-terminal descriptions, and name simply a generalized 2-terminal representation. In this context, observe that amplifier's description that relates its output voltage with its input voltage - without involving in this description amplifier's output and input currents - can be viewed as a type of a generalized 2-terminal circuit element representation. So, such an amplifier model is one of the forms the generalized 2-terminal device can assume.

In this paper, we assume that terminal, port or internal variables occurring in the set of constitutive equations describing a weakly nonlinear amplifier are related to the input signal applied to the whole circuit through Volterra series. Furthermore, we assume here that these Volterra series can be truncated to the first three components. In other words, we assume that neglecting components of orders higher than three in all of the above series do not influence significantly accuracy of the results obtained. By the way, note also that fulfillment of the above assumption can be used as another 
definition of the notion of a weakly (mildly) nonlinear circuit (amplifier); it is of course closely related to that presented just before, however, not identical with.

The set of constitutive equations describing a weakly nonlinear amplifier in the time domain builds the basis for development of amplifier's so-called in-network and inputoutput representations (in the aforementioned domain). On this occasion, note that the existence in fact of two kinds of descriptions for nonlinear circuit elements in nonlinear analysis using Volterrra series (the same regards also nonlinear analysis exploiting phasors) has been pointed out for the first time in [7]; obviously - for linear elements - these descriptions are identical. In [7], they have been named in-network and input-output descriptions.

In derivation of the in-network representation (model) of a weakly nonlinear circuit element, being a part of a larger circuit, one assumes that the input signal is not applied directly to a terminal (or port) of this element. Opposite to this, in derivation of its input-output representation, one assumes that the input signal is applied directly to one of its terminals (or ports). Obviously, these two circuit or circuit element descriptions differ from each other, see for example [7]; this fact will be also evident in the course of this paper.

Here, we derive first generic formulas in the time domain for the aforementioned descriptions, valid for any real or equivalent two-terminal nonlinear circuit element and also for two-terminal equivalents of whole circuits consisting of linear and nonlinear elements. Afterwards, these formulas are transferred into the multi-frequency domains using the multidimensional Fourier transforms [8], [9]. Further analyses are performed exclusively in the multi-frequency domains, which are, as well known, easier to carry out. And at the first instance, using the above generic formulas for elements that are the nonlinear Rosenstark amplifier model components, we derive its in-network and input-output descriptions. Next, we show that the amplifier's input-output representation provides immediately its so-called nonlinear transfer functions of the first (linear), second, and third order - named in such a way, for example, in [8]-[11]. These nonlinear transfer functions specialized for harmonic distortion analysis [12] are identical with the so-called nonlinear coefficients [3], [5], [13], which one obtains in the nonlinear analysis using phasors. The above fact has been pointed out for the first time in [14].

In other words, the nonlinear transfer functions used in harmonic distortion analysis can be viewed as being functions of a single frequency, as is the case in symbolic analysis presented in [3], [5], [13]. Furthermore, as it has been said above, these transfer functions are then identical with the socalled nonlinear coefficients introduced in [3], [13]. That is nonlinear coefficients are nonlinear transfer functions specialized exclusively for performing harmonic distortion analysis - and nothing more. For calculations of more advanced nonlinear distortion measures as, for example, intermodulation and cross-modulation distortions [15], nonlinear coefficients are useless. In these cases, a more general approach using general forms of nonlinear transfer functions, which are not achievable in symbolic analysis [3], [5], [13], must be used. Such a general framework is presented in this paper.
It is also worth noting that the general formulas of innetwork and input-output representations derived here - for the use according to the simplified convention of modelling all the circuit elements as equivalent two-terminal ones allow a simple calculation of nonlinear transfer functions of any circuit topology, as for example, cascade and feedback structures and their combinations occurring in single-, two-, and three-stage amplifiers analysed intensively in the literature recently [3], [5], [13].

This paper is structured as follows: Generalization of the Rosenstark's linear model of an amplifier to a nonlinear one - exploiting operators in the time domain - is developed in Section 2. Furthermore, in this section, it is shown how to describe the components of the above model as equivalent twoterminal circuit elements. Also, a graph visualizing their connections, which will prove to be very useful in further analysis, is developed. In the next section, the in-network and inputoutput representations of the generic nonlinear two-terminal circuit element described by a truncated Volterra series are derived. Afterwards these representations are transformed into the frequency domain (precisely, multi-frequency domains), and the resulting generic expressions are used in Section 4 to get a general form of the nonlinear Rosenstark's model in this domain. Section 4 is also devoted to the specialization of the nonlinear Rosenstark's model in the frequency domain to harmonic distortion analysis. In Section 5, we present an illustrative but a little bit more advanced example of the usage of the theory developed in derivation of the in-network model of two-stage amplifier as a component of three-stage one. Some remarks on such questionable notions as nonlinear signals, intermodulation nonlinearity, making identity between the transfer function poles and zeros lying on the real axis of the complex plane with the related positive-valued frequencies, and on other ones are presented in Section 6. The next section concludes the paper.

\section{Nonlinear Rosenstark's Model And Nonlinear Equivalent Two-TERMINAL CiRCUIT ElEMENTS}

The linear Rosenstark model of an amplifier [1], [2], [5] has been formulated in the frequency domain, using such notions as transfer functions and Fourier transform. Obviously, using the reverse Fourier transform, this model can put into an equivalent form that exploits linear operators working on continuous time signals. The latter is the basis for generalization undertaken in this section. Simply, we assume now occurrence of nonlinear operators in places of linear ones. Using the version of the Rosenstark's model presented in [5] with the nonlinear voltage controlled current source (NVCCS), the similar notation for signal variables as therein, and naming the operators here similarly as the transfer functions in [5], we write

$$
\left\{\begin{array}{l}
\tilde{x}_{o}(t)=H_{1}\left(x_{s}\right)(t)+H_{F}\left(x_{n}\right)(t) \\
x_{o}(t)=H_{D}\left(x_{s}\right)(t)+H_{2}\left(x_{n}\right)(t) \\
x_{n}(t)=g_{m}\left(\tilde{x}_{o}\right)(t)+g_{o}\left(x_{o}\right)(t)
\end{array}\right.
$$

where $x_{s}(t)$ and $x_{o}(t)$ mean the amplifier's input and output voltage (input and output signal), respectively. Further, $\tilde{x}_{o}(t)$ and $x_{n}(t)$ are the internal signals in the model. More precisely, 


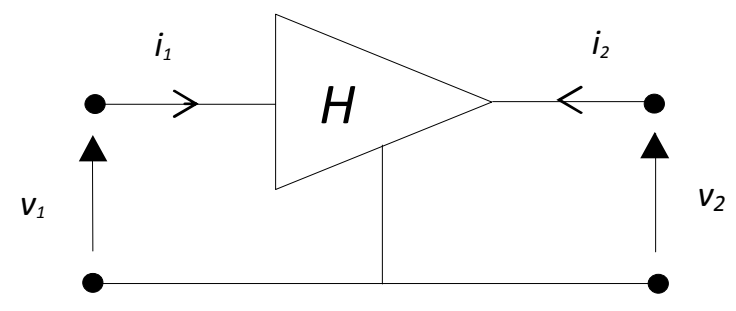

Fig. 1. An amplifier as a two-port.

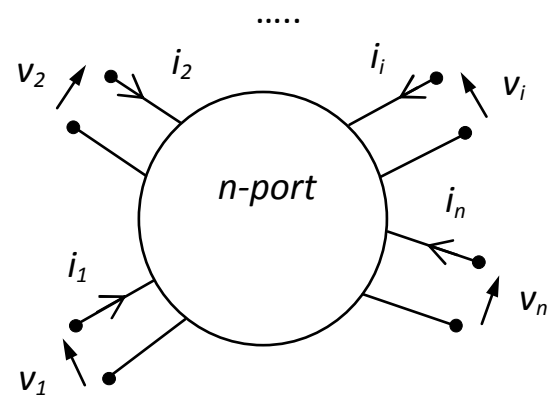

Fig. 2. A scheme of n-port device after [6].

$\tilde{x}_{o}(t)$ means the voltage signal controlling the NVCCS, named here $g_{m}$. But, $x_{n}(t)$ is the current signal being the sum of currents flowing through the parallel connection of $g_{m}$ and nonlinear amplifier's output conductance $g_{o}$. The operators $H_{1}\left(x_{s}\right)(t), H_{F}\left(x_{n}\right)(t), H_{D}\left(x_{s}\right)(t)$, and $H_{2}\left(x_{n}\right)(t)$, in general being nonlinear in this model, describe transmissions of the signals: input $x_{s}(t)$ to internal $\tilde{x}_{o}(t)$, internal $x_{n}(t)$ to internal $\tilde{x}_{o}(t)$, input $x_{s}(t)$ to output $x_{o}(t)$, and internal $x_{n}(t)$ to output $x_{o}(t)$, respectively. For more explanation regarding the choice of the above notation, see [5]. Moreover, note also that a more convenient way of writing operators in (1) was chosen, namely, $H_{1}\left(x_{s}\right)(t)$ instead of $H_{1}\left(x_{s}(t)\right)$ as used usually, and so on.

In [6], Chua has introduced to the circuit theory literature the so-called constitutive relations describing such the circuit components as two-terminal elements and, generally, multiterminal elements, and multi-ports (n-ports). Further, note that a nonlinear amplifier can be viewed as a 2-port, as shown in Fig. 1. Then, it is described by two sets: the first consisting of voltage and current of port 1 (input port) and the second of the same variables but now for port 2 (output port); that is by $\left\{v_{1}, i_{1}\right\}$ and $\left\{v_{2}, i_{2}\right\}$, together with a set of algebraic and non-algebraic relations holding between the elements of the above two sets. In Chua's terminology [6], these relations are called constitutive relations (equations); for more details regarding the subject of constitutive relations of multi-ports see [6], Section III therein.

The set of equations (1) builds a set of the constitutive equations of the nonlinear Rosenstark's model of an amplifier. This set is however specific and has a simplified form. To see this, compare first the description (1) with the port variables in Fig. 1, identifying $x_{s}(t)=v_{1}(t)$ and $x_{o}(t)=v_{2}(t)$. Further, observe the absence of the second variables (signals) in the pairs of port variables, that is of $i_{1}(t)$ and $i_{2}(t)$, in (1). In this respect, we have to do with simplification of the model.

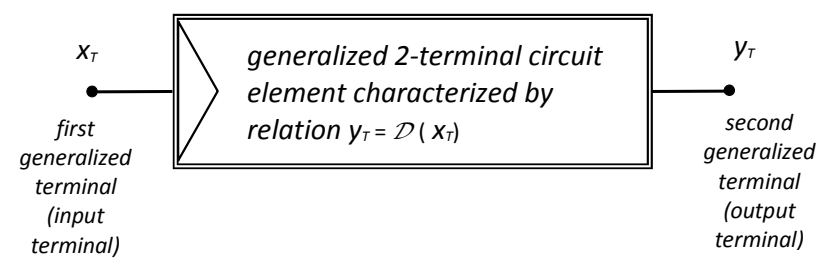

Fig. 3. Symbol and notation used for an equivalent 2-terminal circuit element.

Moreover, note that the variables (signals) $\tilde{x}_{o}(t)$ and $x_{n}(t)$ occurring in (1) are absent in Fig. 1. So the 2-port description by (1) possesses also two internal variables.

Let us now introduce a new notion: of that we call here a 2-port-like-2-terminal or block-like-2-terminal, or simply a generalized 2-terminal circuit element. We use this generic element to model any 2-port or multi-port (circuit block) by an equivalent 2-terminal element. To formulate the definition a generalized 2-terminal circuit element, consider now a scheme of a n-port device as sketched in Fig. 2.

And, let us take into account any two port variables occurring in Fig. 2. We denote them as $x_{T}$ and $y_{T}$, and assume that a relation between them (if such one exists) describes an equivalent 2-terminal circuit element that can be used instead of the n-port device shown in Fig. 2. In other words, from the point of view of the pair $x_{T}$ and $y_{T}$ of the n-port variables, the n-port of Fig. 2 can be equivalently replaced by a 2-terminal circuit element having terminal variables equal to $x_{T}$ and $y_{T}$, respectively. Furthermore, we assume that the variable $x_{T}$ or $y_{T}$ can be any port voltage or port current shown in Fig. 2, and any combination of them (any pair of them) is allowable. That is we can pair, for example, $x_{T}=i_{1}$ with $y_{T}=v_{1}$, or $x_{T}=v_{1}$ with $y_{T}=v_{2}$, or $x_{T}=i_{1}$ with $y_{T}=i_{i}$, or $x_{T}=i_{i}$ with $y_{T}=v_{1}$, or $x_{T}=v_{2}$ with $y_{T}=i_{1}$, and so on. Moreover, in these combinations, $x_{T}$ and $y_{T}$ can assume the meaning of current as well as of voltage. Further, because of the above two properties, such an equivalent 2-terminal circuit element is named here a generalized one. Depending upon the meaning of its terminal variables (current, voltage), it can have a character of a resistor (more general, of an impedance), of a voltage controlled voltage source, and so on.

The above descriptive definition of a circuit element, which we named here the generalized equivalent 2-terminal circuit element, is illustrated in Fig. 3.

The circuit element shown in Fig. 3 possesses two terminals we called generalized ones because of the fact that they are not true terminals when $x_{T}$ and/or $y_{T}$ mean voltages. In these cases, they are physically ports. Furthermore, the above terminals are numbered: the first and the second generalized one, suggesting their different roles in applications discussed further in this paper. In fact, we assume here that the generalized equivalent 2-terminal circuit element plays a role of a signal (terminal variable) processing device from the first to the second generalized terminal. Hence, these nodes in Fig. 3 are also named the input and output terminal, respectively. Further, the direction of the signal transfer is depicted by an arrow inside the element symbol (in Fig. 3). Moreover, we assume that only the signal $y_{T}$ (not $x_{T}$ ) can be an input 


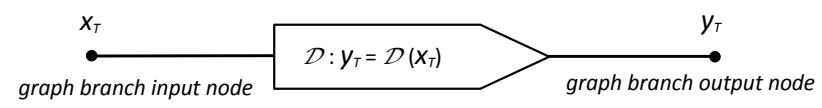

Fig. 4. A generalized 2-terminal circuit element interpreted as a branch of an oriented graph.

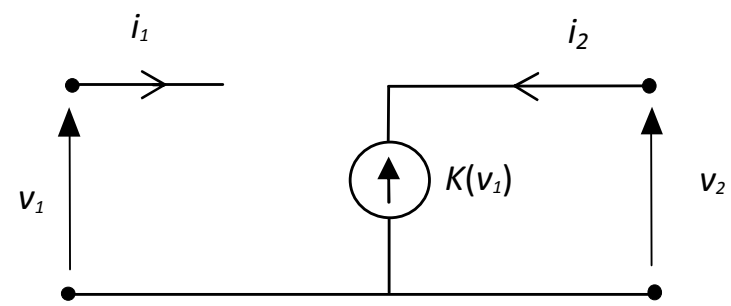

Fig. 5. A scheme of an ideal nonlinear voltage controlled voltage source (NVCVS) as a 2-port.

signal of the following (if any) equivalent 2-terminal circuit element connected to that in Fig. 3. And, the relation existing between the terminal variables $x_{T}$ and $y_{T}$ is expressed as $y_{T}=\mathcal{D}\left(x_{T}\right)$, where $\mathcal{D}$ means, generally, a nonlinear operator. It is assumed that this operator can possess a memory or be memoryless; further, the relation $y_{T}=\mathcal{D}\left(x_{T}\right)$ is, according to [6], the constitutive equation of the equivalent circuit element visualized in Fig. 3. Note also that in some cases, as for example in the case of $y_{T}=\mathcal{D}\left(x_{T}\right)$ describing a resistor (conductor), we can reverse it using a reverse operator $\mathcal{D}^{-1}$, providing $x_{T}=\mathcal{D}^{-1}\left(y_{T}\right)$, where the roles of $x_{T}$ and $y_{T}$ are interchanged. So, in such cases, we can also use a dual generalized 2-terminal circuit elements, having the direction of the signal transfer reversed. Obviously, a reverse operator $\mathcal{D}^{-1}$ does not exist for all the generalized 2-terminal circuit elements; therefore, not in all of them, the roles of terminal variables can be reversed.

Further, it follows from the above description of the generalized 2-terminal circuit element shown in Fig. 3 that this element can be also well described and in a simpler way using the notions of graph theory. Simply, it can be viewed as a branch of an oriented graph, as sketched in Fig. 4.

Comparison of Figs. 3 and 4 shows that only one essential notational difference exists between them, namely, the terminals of 2-terminal circuit element of Fig. 3 are now, in Fig. 4, called graph branch nodes. Moreover, in this paper, we will distinguish between two kinds of graph nodes: a) such ones in which no algebraic operations are performed and those b) in which some algebraic operations are carried out. Regarding the second kind, we will use here exclusively nodes in which summation operation on the incoming signals is performed.

Let us return now to the problem of replacement of the n-port device of Fig. 2 by its equivalent in the form of a generalized 2-terminal circuit element (2-port-like-2-terminal device) visualized in Fig. 3 and ask about the accuracy of such replacement. Here, we sketch only the problem. And, to this end, consider an ideal nonlinear voltage controlled voltage source (NVCVS) of which equivalent circuit is shown in Fig. 5.

It is evident from Fig. 5 that the relation between the following port variables: $v_{1}=x_{T}$ and $v_{2}=y_{T}$ is given by
$v_{2}=K\left(v_{1}\right)$, where $\mathrm{K}$ stands for a nonlinear operator. And, observe that this relation involves only two port variables of the NVCVS as a 2-port. That means it is a perfect one for the purpose of describing the NVCVS in Fig. 5 by an equivalent generalized 2-terminal circuit element. In other words, this equivalent description will be accurate.

Consider now two nonideal nonlinear controlled sources: nonideal NVCCS and nonideal NVCVS of which schemes are depicted in Fig. 6.

Equations relating the port variables in the nonideal NVCCS of Fig. 6(a) and in the nonideal NVCVS of Fig. 6(b) have the following forms

$$
i_{2}=H\left(v_{1}\right)+G_{o} \cdot v_{2} \quad \text { and } \quad v_{2}=K\left(v_{1}\right)+R_{o} \cdot i_{2}
$$

respectively. Observe that in both the cases three port variables are involved, but we would await only two to be able to formulate equivalents of the above 2-ports in forms of generalized 2-terminal circuit elements. We can achieve this approximately by letting $G_{o} \rightarrow 0$ and $R_{o} \rightarrow 0$ in (2). This leads to getting $i_{2} \cong H\left(v_{1}\right)$ and $v_{2} \cong K\left(v_{1}\right)$, respectively. So, in other words, neglecting the loading effects caused by shunt output conductance in Fig. 6(a) and series output resistance in Fig. 6(b), we can replace the nonideal NVCCS and NVCVS by the corresponding generalized 2-terminal circuit elements of the ideal NVCCS and NVCVS (not in sense of an exact replacement, but of an approximate one).

Let us now try to interpret the set of equations (1) in view of the remarks made above with regard to the notion of generalized 2-terminal circuit element. To this end, we recall the fact already pointed out in the explanation beneath Fig. 1 that this description involves only two port variables of an amplifier modeled as a 2-port. Hence, it has an accurate equivalent representation in form of a generalized 2-terminal circuit element. (Caution: Do not misunderstand accuracy of the equivalence between the above representations with the accuracy of modeling a real amplifier by Rosenstark model.) And this equivalent 2-terminal element can be visualized as sketched in Figs. 3 and 4; in further problem analyses and illustrations, we will prefer to use rather the second type of visualization, graph-oriented. In this convention, the amplifier $H$ of Fig. 1, which has the constitutive equation $x_{o}=v_{2}=H\left(v_{1}=x_{s}\right)$ implicitly defined by the set of equations (1), is presented in Fig. 7.

Note that using the same arguments as above, we can interpret similarly all the operators (components) occurring in equations (1): $H_{1}, H_{F}, H_{D}, H_{2}, g_{m}$, and $g_{o}$ as representing accurate equivalent generalized 2-terminal circuit elements (or 2-node oriented graphs). Their equivalent 2-node oriented graphs are summarized in Fig. 8.

Physical meaning (voltage or current) of the graph node (terminal) variables $x_{T}$ and $y_{T}$ is depicted in Fig. 8 for each of the equivalent 2-node oriented graphs. This information will be necessary in building the whole graph of an amplifier described by equations (1) to take care of not-connecting to each other nodes being of different physical type (shortly, to avoid connection of "current nodes" with "voltage nodes").

Now, using the equivalent 2-node oriented graphs of Fig. 8, we can visualize equations (1) as shown in Fig. 9. 


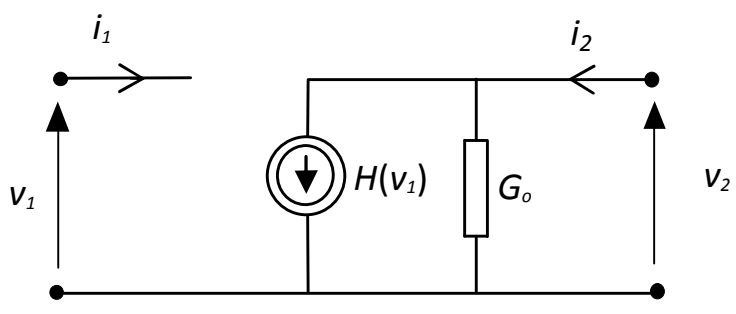

(a)

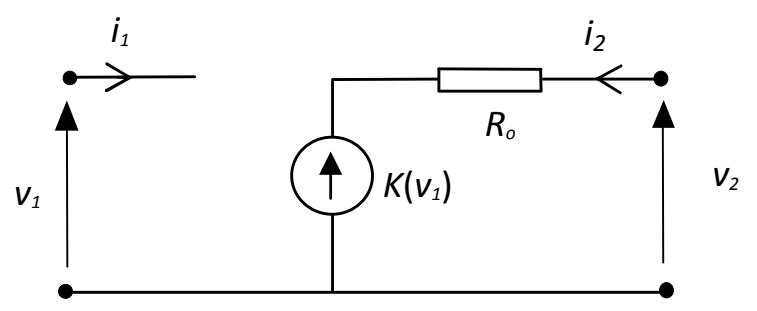

(b)

Fig. 6. (a) A scheme of nonideal NVCCS as a 2-port and, similarly, (b) a scheme of nonideal NVCVS as a 2-port.

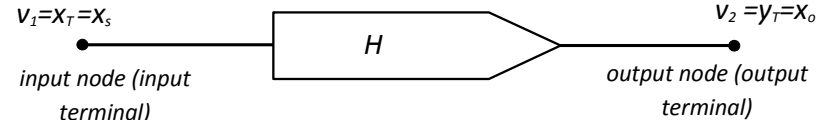

Fig. 7. An equivalent generalized 2-terminal circuit element of a Rosenstark model of an amplifier interpreted as a 2-node oriented graph.

For better understanding of the amplifier graph of Fig. 9, relations between notations used for the variables occurring in equations (1) and those used in Fig. 8 are explicitly depicted in Fig. 9. Moreover, we draw the reader's attention to occurrence of the nodes of two types in Fig. 9: usual ones depicted by dots and the summation nodes. The second type of graph nodes follows from the physical meaning of variables and governing rules associated with them: the first and second Kirchhoff's laws. In other words, there are points in Fig. 9, in which we must add to currents or voltages (according to the above laws).

\section{IN-NETWORK AND INPUT-OUTPUT REPRESENTATIONS of Generic Nonlinear Two-Terminal Circuit ELEMENT DESCRIBED By A TRUNCATEd VolTERRA SERIES}

In this paper, we assume that the relation $y_{T}=\mathcal{D}\left(x_{T}\right)$ describing a generic element we introduced here and called the generalized 2-terminal circuit element can be expanded in a Volterra series [8]-[11]. That is as

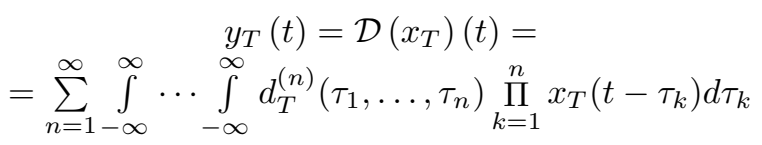

where $d_{T}^{(n)}\left(\tau_{1}, \ldots, \tau_{n}\right)$ means a nonlinear impulse response of the $n$-th order of the generalized 2-terminal circuit element. Observe also that the responses $d_{T}^{(n)}\left(\tau_{1}, \ldots, \tau_{n}\right), n=$ $1,2,3, \ldots$, in (3) can describe the following types of signal transferring: current-to-current, current-to-voltage, voltage-tocurrent, and voltage-to-voltage in case of our generic 2terminal element. Moreover, note that in a particular case of a generic 2-terminal element representing a linear element, (3) reduces to one component, being a standard convolution integral. Also, note that for memoryless generic elements the description in form of 3 becomes a Taylor series.

Further, we restrict ourselves here to consideration of such nonlinear systems (circuits), as in [3], [5], [7], [8], [12]-[20], which are only weakly (mildly) nonlinear. And, it is worth noting at this point that this class of systems has been defined in the literature cited above using different approaches and formulations. In terms of the Volterra series, saying shortly, it encompasses such systems of which analysis with the use of the truncated Volterra series (truncated to the first three terms), suffices. More precisely, in this paper, we understand the aforementioned class as: First, as such one in which descriptions of all the constitutive equations of system's composing elements, and of all the relations between terminal (port, or generalized) variables (signals) and the system's input variable (signal) are expressed in form of Volterra series truncated to the first three terms. And second, each analysis of a system belonging to this class provides results not differing or differing only slightly from those one gets in measurements. Hence, it follows that we assume here

$$
\begin{gathered}
y_{T}(t)=\mathcal{D}\left(x_{T}\right)(t) \cong \int_{-\infty}^{\infty} d_{T}^{(1)}(\tau) x_{T}(t-\tau) d \tau+ \\
+\int_{-\infty}^{\infty} \int_{-\infty}^{\infty} d_{T}^{(2)}\left(\tau_{1}, \tau_{2}\right) x_{T}\left(t-\tau_{1}\right) x_{T}\left(t-\tau_{2}\right) d \tau_{1} d \tau_{2}+ \\
+\int_{-\infty}^{\infty} \int_{-\infty}^{\infty} \int_{-\infty}^{\infty} d_{T}^{(3)}\left(\tau_{1}, \tau_{2}, \tau_{3}\right) x_{T}\left(t-\tau_{1}\right) \times \\
\quad \times x_{T}\left(t-\tau_{2}\right) x_{T}\left(t-\tau_{3}\right) d \tau_{1} d \tau_{2} d \tau_{3}
\end{gathered}
$$

for any generalized 2-terminal element, and the following

$$
\begin{gathered}
y_{T}(t)=y_{T}^{(1)}(t)+y_{T}^{(2)}(t)+y_{T}^{(3)}(t)+\ldots \cong \\
\cong \int_{-\infty}^{\infty} h_{T o}^{(1)}(\tau) x_{s}(t-\tau) d \tau+ \\
+\int_{-\infty}^{\infty} \int_{-\infty}^{\infty} h_{T o}^{(2)}\left(\tau_{1}, \tau_{2}\right) x_{s}\left(t-\tau_{1}\right) x_{s}\left(t-\tau_{2}\right) d \tau_{1} d \tau_{2}+ \\
+\int_{-\infty}^{\infty} \int_{-\infty}^{\infty} \int_{-\infty}^{\infty} h_{T o}^{(3)}\left(\tau_{1}, \tau_{2}, \tau_{3}\right) x_{s}\left(t-\tau_{1}\right) \times \\
\quad \times x_{s}\left(t-\tau_{2}\right) x_{s}\left(t-\tau_{3}\right) d \tau_{1} d \tau_{2} d \tau_{3}
\end{gathered}
$$

and

$$
\begin{gathered}
x_{T}(t)=x_{T}^{(1)}(t)+x_{T}^{(2)}(t)+x_{T}^{(3)}(t)+\ldots \cong \\
\cong \int_{-\infty}^{\infty} h_{T i}^{(1)}(\tau) x_{s}(t-\tau) d \tau+ \\
+\int_{-\infty}^{\infty} \int_{-\infty}^{\infty} h_{T i}^{(2)}\left(\tau_{1}, \tau_{2}\right) x_{s}\left(t-\tau_{1}\right) x_{s}\left(t-\tau_{2}\right) d \tau_{1} d \tau_{2}+ \\
+\int_{-\infty}^{\infty} \int_{-\infty}^{\infty} \int_{-\infty}^{\infty} h_{T i}^{(3)}\left(\tau_{1}, \tau_{2}, \tau_{3}\right) x_{s}\left(t-\tau_{1}\right) \times \\
\quad \times x_{s}\left(t-\tau_{2}\right) x_{s}\left(t-\tau_{3}\right) d \tau_{1} d \tau_{2} d \tau_{3}
\end{gathered}
$$

for the relations between the terminal variables (signals) $y_{T}$ and $x_{T}$, and the input signal applied to the whole system input. 


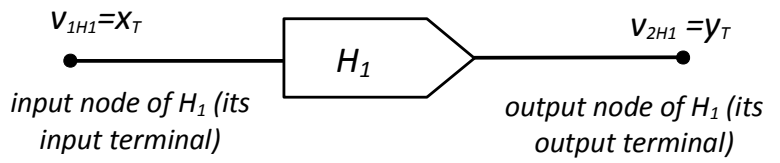

(a)

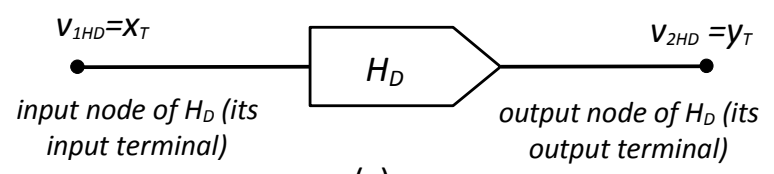

(c)

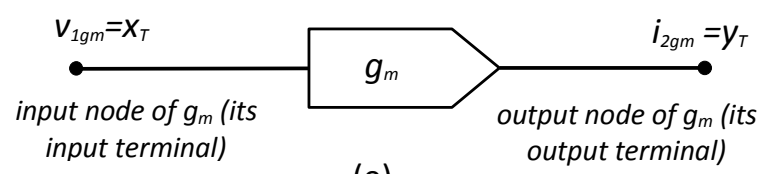

(e)

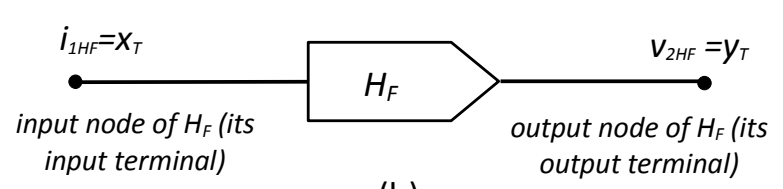

(b)

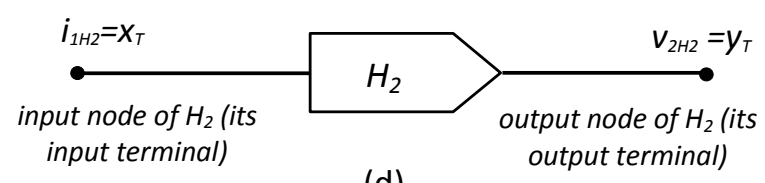

(d)

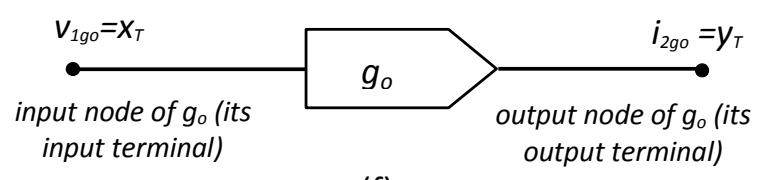

(f)

Fig. 8. Equivalent 2-node oriented graphs (equivalents of the corresponding generalized 2-terminal circuit elements) for the operators occurring in equations (1): (a) for $H_{1}$, (b) for $H_{F}$, (c) for $H_{D}$, (d) for $H_{2}$, (e) for $g_{m}$, and (f) for $g_{o}$.

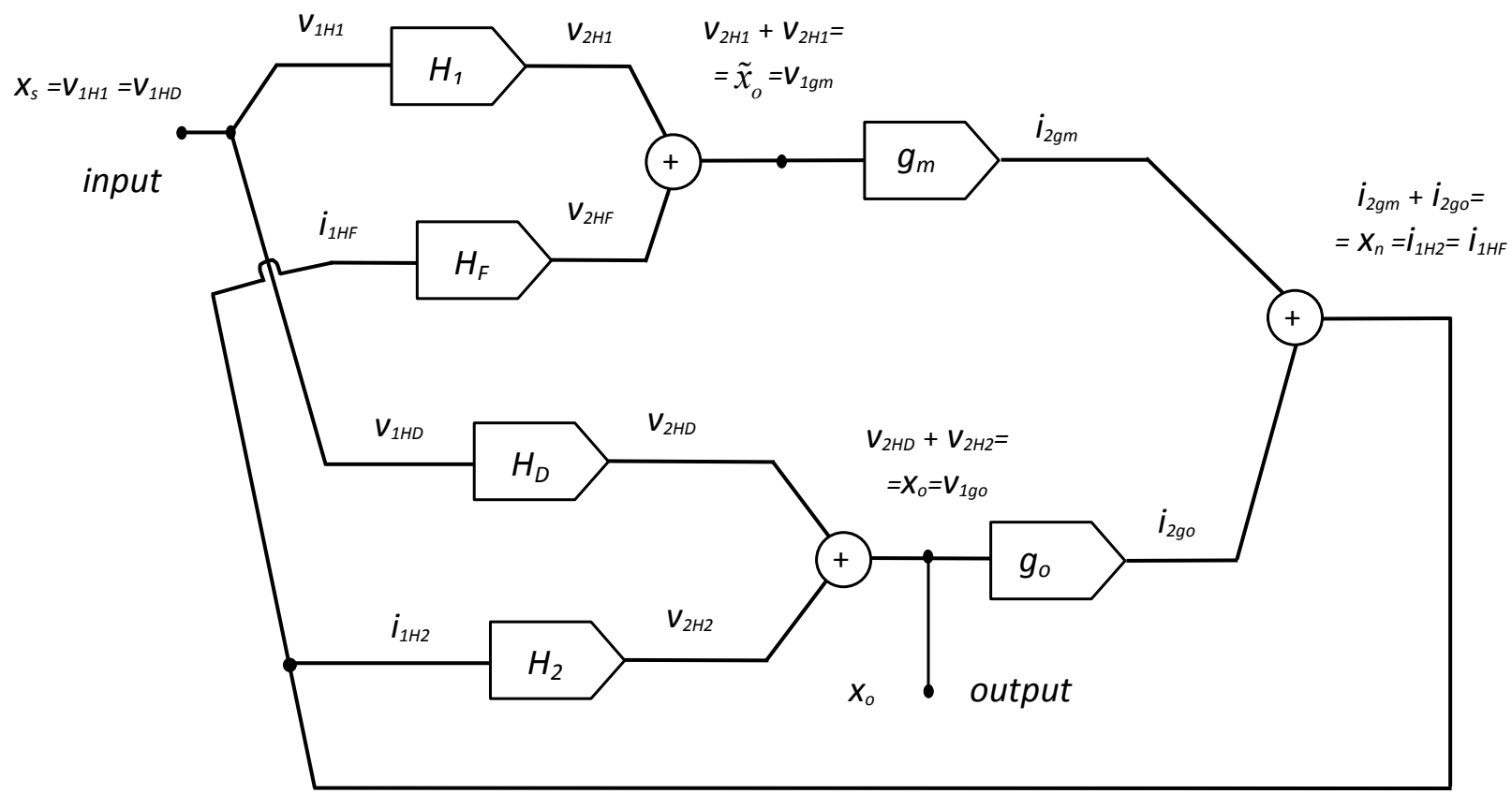

Fig. 9. Oriented graph of the amplifier described by equations (1), constructed using equivalent 2-node graphs of operators $H_{1}, H_{F}, H_{D}, H_{2}, g_{m}$, and $g_{o}$ illustrated in Fig. 8. 
In $(5 a)$ and $(5 b)$, the latter one is denoted as $x_{s}(t)$. Moreover, therein, the corresponding functions $h_{T o}^{(n)}\left(\tau_{1}, \ldots, \tau_{n}\right)$ and $h_{T i}^{(n)}\left(\tau_{1}, \ldots, \tau_{n}\right)$ mean the nonlinear impulse responses of the $n$-th order, $n=1,2,3, \ldots$, of the system's paths: from the system's input to the output and input terminal of the element considered, respectively. Furthermore, $y_{T}^{(n)}(t)$ and $x_{T}^{(n)}(t)$, $n=1,2,3, \ldots$, are used to denote shortly the partial responses in the generic element output response $y_{T}(t)$ and its input response $x_{T}(t)$, accordingly, that is the corresponding one-, two-, and three-dimensional convolution integrals occurring on the right-hand sides of (5a) and (5b), and the next ones. In this paper, we will use the above notion of partial responses to the aforementioned $\mathrm{n}$-dimensional convolution integrals exclusively when they are calculated with respect to the signal $x_{s}(t)$.

It has been shown in [7] and [16] that two different descriptions of composing nonlinear elements (blocks) of weakly nonlinear systems are needed in their analysis. These representations for a given system's composing element depend upon whether an input signal applied to the whole system is identical or not with the input variable of the element considered. They have been called the input-output and in-network description, respectively.

So, to derive the first one for our generic generalized 2-terminal circuit element, we apply $x_{T}(t)=x_{s}(t)$. Taking this into account in $(5 b)$ gives

$$
\begin{gathered}
x_{T}^{(1)}(t)=\int_{-\infty}^{\infty} \delta(\tau) x_{s}(t-\tau) d \tau=x_{s}(t), \\
x_{T}^{(2)}(t)=\int_{-\infty}^{\infty} \int_{-\infty}^{\infty} 0 \cdot x_{s}\left(t-\tau_{1}\right) x_{s}\left(t-\tau_{2}\right) d \tau_{1} d \tau_{2}=0, \\
x_{T}^{(3)}(t)=\int_{-\infty}^{\infty} \int_{-\infty}^{\infty} \int_{-\infty}^{\infty} 0 \cdot x_{s}\left(t-\tau_{1}\right) x_{s}\left(t-\tau_{2}\right) \times \\
\times x_{s}\left(t-\tau_{3}\right) d \tau_{1} d \tau_{2} d \tau_{3}=0
\end{gathered}
$$

and

$$
\begin{gathered}
h_{T i}^{(1)}(t)=\delta(t), \quad h_{T i}^{(2)}\left(t_{1}, t_{2}\right)=0, \\
h_{T i}^{(3)}\left(t_{1}, t_{2}, t_{3}\right)=0
\end{gathered}
$$

where $\delta(t)$ means the Dirac impulse, and $t_{1}, t_{2}, t_{3}$ are the auxiliary time variables. Further, substitution of $x_{T}(t)=x_{s}(t)$ in (4) and comparison with (5a) results in

$$
\begin{gathered}
y_{T}^{(1)}(t)=\int_{-\infty}^{\infty} d_{T}^{(1)}(\tau) x_{s}(t-\tau) d \tau \\
y_{T}^{(2)}(t)=\int_{-\infty}^{\infty} \int_{-\infty}^{\infty} d_{T}^{(2)}\left(\tau_{1}, \tau_{2}\right) x_{s}\left(t-\tau_{1}\right) \times \\
y_{T}^{(3)}(t)=\int_{-\infty}^{\infty} \int_{-\infty}^{\infty} \int_{-\infty}^{\infty} d_{T}^{(3)}\left(\tau_{1}, \tau_{2}, \tau_{3}\right) x_{s}\left(t-\tau_{1}\right) \times \\
\times x_{s}\left(t-\tau_{2}\right) x_{s}\left(t-\tau_{3}\right) d \tau_{1} d \tau_{2} d \tau_{3}
\end{gathered}
$$

and

$$
\begin{gathered}
h_{T o}^{(1)}(t)=d_{T}^{(1)}(t), h_{T o}^{(2)}\left(t_{1}, t_{2}\right)=d_{T}^{(2)}\left(t_{1}, t_{2}\right), \\
h_{T o}^{(3)}\left(t_{1}, t_{2}, t_{3}\right)=d_{T}^{(3)}\left(t_{1}, t_{2}, t_{3}\right)
\end{gathered}
$$

The relations (7a) and (7b) together with the complementing ones (6a) and (6b) constitute the input-output representation in the time domain of our generic generalized 2-terminal circuit element.

Now, we remember that the case of in-network description is characterized by $x_{T}(t) \neq x_{s}(t)$. So, we get then from (5b)

$$
\begin{gathered}
x_{T r}^{(1)}(t)=\int_{-\infty}^{\infty} h_{T i}^{(1)}(\tau) x_{s}(t-\tau) d \tau, x_{T r}^{(2)}(t)= \\
=\int_{-\infty}^{\infty} \int_{-\infty}^{\infty} h_{T i}^{(2)}\left(\tau_{1}, \tau_{2}\right) x_{s}\left(t-\tau_{1}\right) x_{s}\left(t-\tau_{2}\right) d \tau_{1} d \tau_{2}, \\
x_{T r}^{(3)}(t)=\int_{-\infty}^{\infty} \int_{-\infty}^{\infty} \int_{-\infty}^{\infty} h_{T i}^{(3)}\left(\tau_{1}, \tau_{2}, \tau_{3}\right) x_{s}\left(t-\tau_{1}\right) \times \\
\times x_{s}\left(t-\tau_{2}\right) x_{s}\left(t-\tau_{3}\right) d \tau_{1} d \tau_{2} d \tau_{3}
\end{gathered}
$$

where an additional letter " $r$ " has been added to " $T$ " in subscripts of $x_{T}^{(n)}(t), n=1,2,3$, to distinguish this case from the previous "input-output" one (see (6a) and (6b)).

Similarly, let us denote the corresponding partial output responses in the in-network description of a generic generalized 2-terminal circuit element by $y_{T r}^{(n)}(t), n=1,2,3$, with the additional letter " $r$ " in subscripts. Observe next that (4) and (5a) must give the same result for $y_{T}(t)$, so combining these expressions, we can write

$$
\begin{gathered}
y_{T r}^{(1)}(t)+y_{T r}^{(2)}(t)+y_{T r}^{(3)}(t) \cong \\
\cong \int_{-\infty}^{\infty} d_{T}^{(1)}(\tau) x_{T r}(t-\tau) d \tau+ \\
\int_{-\infty}^{\infty} \int_{-\infty}^{\infty} d_{T}^{(2)}\left(\tau_{1}, \tau_{2}\right) x_{T r}\left(t-\tau_{1}\right) x_{T r}\left(t-\tau_{2}\right) d \tau_{1} d \tau_{2}+ \\
+\int_{-\infty}^{\infty} \int_{-\infty}^{\infty} \int_{-\infty}^{\infty} d_{T}^{(3)}\left(\tau_{1}, \tau_{2}, \tau_{3}\right) x_{T r}\left(t-\tau_{1}\right) \times \\
\times x_{T r}\left(t-\tau_{2}\right) x_{T r}\left(t-\tau_{3}\right) d \tau_{1} d \tau_{2} d \tau_{3}
\end{gathered}
$$

Applying then $x_{T r}(t) \cong x_{T r}^{(1)}(t)+x_{T r}^{(2)}(t)+x_{T r}^{(3)}(t)$, which follows from $(5 b)$, in (9a) gives

$$
\begin{gathered}
y_{T r}^{(1)}(t)+y_{T r}^{(2)}(t)+y_{T r}^{(3)}(t) \cong \\
\cong \int_{-\infty}^{\infty} d_{T}^{(1)}(\tau) x_{T r}^{(1)}(t-\tau) d \tau+ \\
+\int_{-\infty}^{\infty} d_{T}^{(1)}(\tau) x_{T r}^{(2)}(t-\tau) d \tau+ \\
+\int_{-\infty}^{\infty} d_{T}^{(1)}(\tau) x_{T r}^{(3)}(t-\tau) d \tau+ \\
\int_{-\infty}^{\infty} \int_{-\infty}^{\infty} d_{T}^{(2)}\left(\tau_{1}, \tau_{2}\right) x_{T r}^{(1)}\left(t-\tau_{1}\right) \times \\
+\int_{-\infty}^{\infty} \int_{-\infty}^{\infty} d_{T r}^{(2)}\left(t-\tau_{2}\right) d \tau_{1} d \tau_{2}+ \\
+\int_{-\infty}^{\infty} \int_{-\infty}^{\infty} d_{T r}^{(2)}\left(\tau_{1}^{(1)}\left(t-\tau_{1}\right) \times\right. \\
+\int_{-\infty}^{\infty} \int_{-\infty}^{\infty} \int_{-\infty}^{(2)}\left(t-\tau_{1}\right) \times \\
\quad \times x_{T r}^{(1)}\left(t-\tau_{T}^{(3)}\left(\tau_{2}\right) x_{T r}^{(1)}\left(t-\tau_{2}, \tau_{3}\right) x_{T r}^{(1)}\left(t-\tau_{1}\right) \times\right. \\
+\tau_{1} d \tau_{2} d \tau_{3}
\end{gathered}
$$

Having in mind the relations (5a) and (5b), we can now compare the components of the same order (degree) with 
respect to the input signal $x_{s}(t)$ on both sides of (9b). This will give us the following equations

$$
\begin{gathered}
y_{T r}^{(1)}(t)=\int_{-\infty}^{\infty} d_{T}^{(1)}(\tau) x_{T r}^{(1)}(t-\tau) d \tau \\
y_{T r}^{(2)}(t)=\int_{-\infty}^{\infty} d_{T}^{(1)}(\tau) x_{T r}^{(2)}(t-\tau) d \tau+ \\
+\int_{-\infty}^{\infty} \int_{-\infty}^{\infty} d_{T}^{(2)}\left(\tau_{1}, \tau_{2}\right) x_{T r}^{(1)}\left(t-\tau_{1}\right) \times \\
\times x_{T r}^{(1)}\left(t-\tau_{2}\right) d \tau_{1} d \tau_{2} \\
y_{T r}^{(3)}(t)=\int_{-\infty}^{\infty} d_{T}^{(1)}(\tau) x_{T r}^{(3)}(t-\tau) d \tau+ \\
+\int_{-\infty}^{\infty} \int_{-\infty}^{\infty} d_{T}^{(2)}\left(\tau_{1}, \tau_{2}\right) x_{T r}^{(1)}\left(t-\tau_{1}\right) \times \\
+\int_{-\infty}^{\infty} \int_{-\infty}^{\infty} d_{T}^{(2)}\left(\tau_{1}, \tau_{2}\right) x_{T r}^{(2)}\left(t-\tau_{1}\right) \times \\
+\int_{-\infty}^{\infty} \int_{-\infty}^{\infty} \int_{-\infty}^{\infty} d_{T r}^{(3)}\left(t-\tau_{2}\right) d \tau_{1} d \tau_{2}+ \\
\times x_{T r}^{(1)}\left(t-\tau_{2}, \tau_{3}\right) x_{T r}^{(1)}\left(t-\tau_{1}\right) \times \\
(1)\left(t-\tau_{3}\right) d \tau_{1} d \tau_{2} d \tau_{3}
\end{gathered}
$$

Mathematically rigorous proof of correctness of the procedure applied above in derivation of equations (10a), (10b), and (10c), which uses the properties of homogeneous polynomial mappings, can be found in [16].

The relations (10a), (10b), and (10c) together with (8), the complementing one, constitute the in-network description in the time domain of our generic generalized 2-terminal circuit element.

To transform the expressions derived above in the time domain into the multi-frequency domains, one must introduce some artificial auxiliary time variables in these expressions. For this purpose, a standard procedure is used as described, for instance, in [8], [21]. According to it, we apply here

$$
\begin{gathered}
x_{T r}^{(n)}(t) \rightarrow x_{T r}^{(n)}\left(t_{1}, \ldots, t_{n}\right), \\
y_{T r}^{(n)}(t) \rightarrow y_{T r}^{(n)}\left(t_{1}, \ldots, t_{n}\right)
\end{gathered}
$$

$n=1,2,3$, for the functions on the left-hand sides of equalities in (8) and the partial output responses in (10a)-(10c), respectively, and

$$
\begin{gathered}
x_{s}^{n}(t) \rightarrow x_{s}^{n}\left(t_{1}, \ldots, t_{n}\right)=x_{s}\left(t_{1}\right) \cdots x_{s}\left(t_{n}\right), \\
x_{T r}^{\left(i_{1}\right)}(t) \cdots x_{T r}^{\left(i_{n}\right)}(t) \rightarrow x_{T r}^{\left(i_{1}\right)}\left(t_{1}\right) \cdots x_{T r}^{\left(i_{m}\right)}\left(t_{n}\right),
\end{gathered}
$$

where $i_{1}+\ldots+i_{m}=n$ and $m \leq n$ for the powers of $x_{s}$ and $x_{T r}$ (of different arguments and denoted shortly as seen above), which occur under the integrals on the righthand sides of (8) and (10a)-(10c), respectively. In (11a) and (11b), $t_{1}, \ldots, t_{n}, n=1,2,3$, denote the artificial auxiliary time variables introduced.

The so-called multidimensional ( $n$-dimensional, $n=$ $1,2,3, \ldots)$ Fourier transforms [8], [9] are defined as

$$
\begin{aligned}
& G^{(n)}\left(f_{1}, \ldots, f_{n}\right)=\int_{-\infty}^{\infty} \cdots \int_{-\infty}^{\infty} g^{(n)}\left(t_{1}, \ldots, t_{n}\right) \times \\
& \quad \times \exp \left(-j 2 \pi\left(f_{1} t_{1}+\ldots+f_{n} t_{n}\right)\right) d t_{1} \cdots d t_{n}
\end{aligned}
$$

where $G^{(n)}\left(f_{1}, \ldots, f_{n}\right)$ denotes the $n$-dimensional Fourier transform of a function $g^{(n)}\left(t_{1}, \ldots, t_{n}\right), n=1,2,3, \ldots$, expressed as a function of $n$ arguments being artificial auxiliary time variables. Consequently, $f_{1}, \ldots, f_{n}$ in (12) are the frequencies of the $n$-dimensional frequency space.

By exploiting (12) on both the sides of equalities in (8) and (10a)-(10c), we transfer them into the corresponding multifrequency spaces. The operations, we need to carry out then, are standard; their details have been explained in the literature, for example, see [8], [10] and [11]. Because of this fact, we present only the final results in what follows

$$
\begin{gathered}
X_{T r}^{(1)}(f)=H_{T i}^{(1)}(f) X_{s}(f), \\
X_{T r}^{(2)}\left(f_{1}, f_{2}\right)=H_{T i}^{(2)}\left(f_{1}, f_{2}\right) X_{s}\left(f_{1}\right) X_{s}\left(f_{2}\right), \\
X_{T r}^{(3)}\left(f_{1}, f_{2}, f_{3}\right)=H_{T i}^{(3)}\left(f_{1}, f_{2}, f_{3}\right) \times \\
\times X_{s}\left(f_{1}\right) X_{s}\left(f_{2}\right) X_{s}\left(f_{3}\right) \\
Y_{T r}^{(1)}(f)=D_{T}^{(1)}(f) X_{T r}^{(1)}(f) \\
Y_{T r}^{(2)}\left(f_{1}, f_{2}\right)=D_{T}^{(1)}\left(f_{1}+f_{2}\right) X_{T r}^{(2)}\left(f_{1}, f_{2}\right)+ \\
+D_{T}^{(2)}\left(f_{1}, f_{2}\right) X_{T r}^{(1)}\left(f_{1}\right) X_{T r}^{(1)}\left(f_{2}\right) \\
Y_{T r}^{(3)}\left(f_{1}, f_{2}, f_{3}\right)= \\
D_{T}^{(1)}\left(f_{1}+f_{2}+f_{3}\right) X_{T r}^{(3)}\left(f_{1}, f_{2}, f_{3}\right)+ \\
+D_{T}^{(2)}\left(f_{1}, f_{2}+f_{3}\right) X_{T r}^{(1)}\left(f_{1}\right) X_{T r}^{(2)}\left(f_{2}, f_{3}\right)+ \\
+D_{T}^{(2)}\left(f_{1}+f_{2}, f_{3}\right) X_{T r}^{(2)}\left(f_{1}, f_{2}\right) X_{T r}^{(1)}\left(f_{3}\right)+ \\
+D_{T}^{(3)}\left(f_{1}, f_{2}, f_{3}\right) X_{T r}^{(1)}\left(f_{1}\right) X_{T r}^{(1)}\left(f_{2}\right) X_{T r}^{(1)}\left(f_{3}\right)
\end{gathered}
$$

Note that as mentioned above $f_{1}, f_{2}$, and $f_{3}$ occurring in (13a- $(13 \mathrm{~d})$ represent the frequency variables characterizing the corresponding multidimensional frequency spaces. That is $f_{1}$ (denoted here also by $f$ ) characterizes the one-dimensional frequency space, $f_{1}$ and $f_{2}$ characterize the two-dimensional frequency space, and $f_{1}, f_{2}, f_{3}$ characterize the threedimensional frequency space. Moreover, $D_{T}^{(1)}, H_{T i}^{(1)}, X_{T r}^{(1)}$, $X_{s}, Y_{T r}^{(1)}$, further $D_{T}^{(2)}, H_{T i}^{(2)}, X_{T r}^{(2)}, Y_{T r}^{(2)}$, and finally $D_{T}^{(3)}$, $H_{T i}^{(3)}, X_{T r}^{(3)}, Y_{T r}^{(3)}$ denote the one-, two-, and three-dimensional, respectively, Fourier transforms of the corresponding impulse responses and partial responses of the successive orders, occurring in (13a)-(13d). They depend upon the frequencies $f_{1}$, $f_{2}$, and $f_{3}$ as indicated. Moreover, observe that the functions $D_{T}^{(1)}, D_{T}^{(2)}$, and $D_{T}^{(3)}$ describe the corresponding nonlinear parts (of the first-, second-, and third-order, respectively) of a generic generalized 2-terminal nonlinear circuit element in the one-, two-, and three-dimensional frequency spaces, accordingly. Note also that $H_{T i}^{(1)}, H_{T i}^{(2)}$, and $H_{T i}^{(3)}$ are also called the nonlinear transfer functions of the corresponding orders $n=1,2,3$ (for $n=1$ meaning the standard linear transfer function).

Using the same procedure as above to transfer the relations (6a), (6b), (7a), and (7b) derived for the input-output model 
in the time domain, we get now

$$
\begin{gathered}
X_{T}^{(1)}(f)=1 \cdot X_{s}(f)=X_{s}(f), \\
X_{T}^{(2)}\left(f_{1}, f_{2}\right)=0 \cdot X_{s}\left(f_{1}\right) X_{s}\left(f_{2}\right)=0, \\
X_{T}^{(3)}\left(f_{1}, f_{2}, f_{3}\right)=0 \cdot X_{s}\left(f_{1}\right) X_{s}\left(f_{2}\right) X_{s}\left(f_{3}\right)=0 \\
H_{T i}^{(1)}(f)=1, H_{T i}^{(2)}\left(f_{1}, f_{2}\right)=0, \\
H_{T i}^{(3)}\left(f_{1}, f_{2}, f_{3}\right)=0 \\
Y_{T}^{(2)}\left(f_{1}, f_{2}\right)=D_{T}^{(2)}\left(f_{1}, f_{2}\right) X_{s}\left(f_{1}\right) X_{s}\left(f_{2}\right), \\
Y_{T}^{(3)}\left(f_{1}, f_{2}, f_{3}\right)= \\
=D_{T}^{(3)}\left(f_{1}, f_{2}, f_{3}\right) X_{s}\left(f_{1}\right) X_{s}\left(f_{2}\right) X_{s}\left(f_{3}\right) \\
H_{T o}^{(1)}(f)=D_{T}^{(1)}(f), \\
H_{T o}^{(2)}\left(f_{1}, f_{2}\right)=D_{T}^{(2)}\left(f_{1}, f_{2}\right), \\
H_{T o}^{(3)}\left(f_{1}, f_{2}, f_{3}\right)=D_{T}^{(3)}\left(f_{1}, f_{2}, f_{3}\right)
\end{gathered}
$$

equivalently in the multi-frequency spaces for our generic generalized 2-terminal nonlinear circuit element. Moreover the meaning of the functions occurring in (14a)-(14d) can be deduced similarly as it was done in the detailed explanations beneath (13a)-(13d) for the in-network model.

\section{Nonlinear Rosenstark's Model in the FREQUENCY DOMAIN AND ITS SPECIALIZATION TO HARMONIC DISTORTION ANALYSIS}

In this paper, we understand the nonlinear Rosenstark's model in the frequency domain as such one that has a structure shown in Fig. 9, but all of its composing elements are described now in the multi-frequency spaces according to the rules developed in the previous section. Note further that this structure implies the use of the input-output description developed to the elements depicted as $H_{1}$ and $H_{D}$ in Fig. 9, and the in-network representation to the remaining ones. In what follows, we also simplify our nonlinear Rosenstark's model in a way as it has been done in [5]. That is we assume here that the following four elements: $H_{1}, H_{D}, H_{F}$, and $H_{2}$ in Fig. 9 are linear. Then, only the elements $g_{m}$ and $g_{o}$ remain nonlinear.

The above assumptions lead to simplification of the generic descriptions (14c) and (14d), which are to be used for $H_{1}$ and $H_{D}$, to

$$
\begin{gathered}
Y_{T}^{(1)}(f)=D_{T}^{(1)}(f) X_{s}(f), Y_{T}^{(2)}\left(f_{1}, f_{2}\right)=0, \\
Y_{T}^{(3)}\left(f_{1}, f_{2}, f_{3}\right)=0
\end{gathered}
$$

and

$$
\begin{aligned}
& H_{T o}^{(1)}(f)=D_{T}^{(1)}(f), H_{T o}^{(2)}\left(f_{1}, f_{2}\right)=0 \text {, } \\
& H_{\text {To }}^{(3)}\left(f_{1}, f_{2}, f_{3}\right)=0
\end{aligned}
$$

Moreover, the generic descriptions (13b)-(13d), which are to be used for another two elements: $H_{F}$ and $H_{2}$, reduce in this case to

$$
\begin{gathered}
Y_{T r}^{(1)}(f)=D_{T}^{(1)}(f) X_{T r}^{(1)}(f) \\
Y_{T r}^{(2)}\left(f_{1}, f_{2}\right)=D_{T}^{(1)}\left(f_{1}+f_{2}\right) X_{T r}^{(2)}\left(f_{1}, f_{2}\right) \\
Y_{T r}^{(3)}\left(f_{1}, f_{2}, f_{3}\right)= \\
=D_{T}^{(1)}\left(f_{1}+f_{2}+f_{3}\right) X_{T r}^{(3)}\left(f_{1}, f_{2}, f_{3}\right)
\end{gathered}
$$

Furthermore, assume the descriptions of the elements $g_{m}$ and $g_{o}$ in Fig. 9 in form of the third-order polynomials

$$
\begin{gathered}
i_{2 g m}=g_{m}\left(v_{1 g m}\right)= \\
=g_{m 1} v_{1 g m}+g_{m 2} v_{1 g m}^{2}+g_{m 3} v_{1 g m}^{3}
\end{gathered}
$$

and

$$
i_{2 g o}=g_{o}\left(v_{1 g o}\right)=g_{o 1} v_{1 g o}+g_{o 2} v_{1 g o}^{2}+g_{o 3} v_{1 g o}^{3}
$$

with the coefficients $g_{m 1}, g_{m 2}, g_{m 3}$ and $g_{o 1}, g_{o 2}, g_{o 3}$ being real numbers. The series (17a) and (17b), being the truncated Maclaurin expansions, can be obviously also viewed as the truncated Volterra series discussed in the previous section. More precisely, they are now, in this form, the Volterra series without memory. Hence, because of the above fact, the procedure and expressions derived in Section 3 encompass also this case, allowing us to identify the generic $D_{T}^{(i)}$,s, $n=1,2,3$, for $g_{m}$ and $g_{o}$ needed in the case of $(13 \mathrm{~b})-(13 \mathrm{~d})$. We obtain then

$$
\begin{gathered}
D_{T}^{(1)}(f)=g_{m 1} \text { or } g_{o 1} \\
D_{T}^{(2)}\left(f_{1}, f_{2}\right)=g_{m 2} \text { or } g_{o 2} \\
D_{T}^{(3)}\left(f_{1}, f_{2}, f_{3}\right)=g_{m 3} \text { or } g_{o 3}
\end{gathered}
$$

respectively.

Using now the corresponding generic input-output and innetwork descriptions developed to the network elements of Fig. 9, and taking also into account the simplifications (15a)(15b) and (16a)-(16c) together with the particular results for $g_{m}$ and $g_{o}$, we arrive at the following sets of equations for the whole network of Fig. 9

$$
\left\{\begin{array}{l}
Y_{H 1}^{(1)}(f)+Y_{H F r}^{(1)}(f)=D_{H 1}^{(1)}(f) X_{s}(f)+ \\
+D_{H F}^{(1)}(f) X_{H F r}^{(1)}(f)=X_{g m r}^{(1)}(f) \\
Y_{g m r}^{(1)}(f)=g_{m 1} X_{g m r}^{(1)}(f) \\
Y_{H D}^{(1)}(f)+Y_{H 2 r}^{(1)}(f)=D_{H D}^{(1)}(f) X_{s}(f)+ \\
\quad+D_{H 2}^{(1)}(f) X_{H 2 r}^{(1)}(f)=X_{g o r}^{(1)}(f) \\
Y_{g o r}^{(1)}(f)=g_{o 1} X_{g o r}^{(1)}(f) \\
Y_{g m r}^{(1)}(f)+Y_{g o r}^{(1)}(f)=X_{H F r}^{(1)}(f)=X_{H 2 r}^{(1)}(f)
\end{array}\right.
$$




$$
\begin{aligned}
& \left\{\begin{array}{l}
Y_{H 1}^{(2)}\left(f_{1}, f_{2}\right)+Y_{H F r}^{(2)}\left(f_{1}, f_{2}\right)= \\
\quad=0+D_{H F}^{(1)}\left(f_{1}+f_{2}\right) X_{H F r}^{(2)}\left(f_{1}, f_{2}\right)= \\
=X_{g m r}^{(2)}\left(f_{1}, f_{2}\right) \\
Y_{g m r}^{(2)}\left(f_{1}, f_{2}\right)=g_{m 1} X_{g m r}^{(2)}\left(f_{1}, f_{2}\right)+ \\
\quad+g_{m 2} X_{g m r}^{(1)}\left(f_{1}\right) X_{g m r}^{(1)}\left(f_{2}\right) \\
Y_{H D}^{(2)}\left(f_{1}, f_{2}\right)+Y_{H 2 r}^{(2)}\left(f_{1}, f_{2}\right)= \\
=0+D_{H 2}^{(1)}\left(f_{1}+f_{2}\right) X_{H 2 r}^{(2)}\left(f_{1}, f_{2}\right)= \\
\quad=X_{\text {gor }}^{(2)}\left(f_{1}, f_{2}\right) \\
Y_{\text {gor }}^{(2)}\left(f_{1}, f_{2}\right)=g_{o 1} X_{\text {gor }}^{(2)}\left(f_{1}, f_{2}\right)+ \\
\quad+g_{o 2} X_{\text {gor }}^{(1)}\left(f_{1}\right) X_{\text {gor }}^{(1)}\left(f_{2}\right) \\
Y_{\text {gmr }}^{(2)}\left(f_{1}, f_{2}\right)+Y_{\text {gor }}^{(2)}\left(f_{1}, f_{2}\right)= \\
=X_{H F r}^{(2)}\left(f_{1}, f_{2}\right)=X_{H 2 r}^{(2)}\left(f_{1}, f_{2}\right)
\end{array}\right. \\
& \left\{\begin{array}{c}
Y_{H 1}^{(3)}\left(f_{1}, f_{2}, f_{3}\right)+Y_{H F r}^{(3)}\left(f_{1}, f_{2}, f_{3}\right)= \\
=0+D_{H F}^{(1)}\left(f_{1}+f_{2}+f_{3}\right) \times \\
\quad \times X_{H F r}^{(3)}\left(f_{1}, f_{2}, f_{3}\right)=X_{g m r}^{(3)}\left(f_{1}, f_{2}, f_{3}\right) \\
Y_{g m r}^{(3)}\left(f_{1}, f_{2}, f_{3}\right)=g_{m 1} X_{g m r}^{(3)}\left(f_{1}, f_{2}, f_{3}\right)+ \\
\quad+g_{m 2} X_{g m r}^{(1)}\left(f_{1}\right) X_{g m r}^{(2)}\left(f_{2}, f_{3}\right)+ \\
\quad+g_{m 2} X_{g m r}^{(2)}\left(f_{1}, f_{2}\right) X_{g m r}^{(1)}\left(f_{3}\right)+ \\
\quad+g_{m 3} X_{g m r}^{(1)}\left(f_{1}\right) X_{\text {gmr }}^{(1)}\left(f_{2}\right) X_{g m r}^{(1)}\left(f_{3}\right) \\
Y_{H D}^{(3)}\left(f_{1}, f_{2}, f_{3}\right)+Y_{H 2 r}^{(3)}\left(f_{1}, f_{2}, f_{3}\right)= \\
\quad=0+D_{H 2}^{(1)}\left(f_{1}+f_{2}+f_{3}\right) \times \\
\quad \times X_{H 2 r}^{(3)}\left(f_{1}, f_{2}, f_{3}\right)=X_{\text {gor }}^{(3)}\left(f_{1}, f_{2}, f_{3}\right) \\
Y_{\text {gor }}^{(3)}\left(f_{1}, f_{2}, f_{3}\right)=g_{o 1} X_{\text {gor }}^{(3)}\left(f_{1}, f_{2}, f_{3}\right)+ \\
\quad+g_{o 2} X_{\text {gor }}^{(1)}\left(f_{1}\right) X_{\text {gor }}^{(2)}\left(f_{2}, f_{3}\right)+ \\
\quad+g_{o 2} X_{\text {gor }}^{(2)}\left(f_{1}, f_{2}\right) X_{\text {gor }}^{(1)}\left(f_{3}\right)+ \\
\quad+g_{o 3} X_{\text {gor }}^{(1)}\left(f_{1}\right) X_{\text {gor }}^{(1)}\left(f_{2}\right) X_{\text {gor }}^{(1)}\left(f_{3}\right) \\
Y_{\text {gmr }}^{(3)}\left(f_{1}, f_{2}, f_{3}\right)+Y_{\text {gor }}^{(3)}\left(f_{1}, f_{2}, f_{3}\right)= \\
\quad=X_{H F r}^{(3)}\left(f_{1}, f_{2}, f_{3}\right)=X_{H 2 r}^{(3)}\left(f_{1}, f_{2}, f_{3}\right)
\end{array}\right.
\end{aligned}
$$

Note that equations (19), (20), and (21) regard, respectively, the amplifier first-, second-, and third-order partial responses. Moreover, observe that in writing the aforementioned equations, there was no need to use the relations (13a) of the in-network description - only the relations (13b)-(13d) were exploited. Further, the generic subscript " $T$ " assumed the meaning of: $H 1, H F, H D, H 2, g m$, and $g o$ (for $H_{1}, \ldots$, and so on) at the corresponding places.

We can now solve the sets of equations (19), (20), and (21) to obtain successively expressions for
$X_{\text {gor }}^{(1)}(f) / X_{s}(f), \quad X_{\text {gor }}^{(2)}\left(f_{1}, f_{2}\right) /\left[X_{s}\left(f_{1}\right) X_{s}\left(f_{2}\right)\right], \quad$ and $X_{\text {gor }}^{(3)}\left(f_{1}, f_{2}, f_{3}\right) /\left[X_{s}\left(f_{1}\right) X_{s}\left(f_{2}\right) X_{s}\left(f_{3}\right)\right]$. These ratios, according to the input-output description of a generic circuit element by $(14 \mathrm{c})$, are the nonlinear transfer functions of the corresponding orders (first, second, and third one) of the whole amplifier of Fig. 9. In our terminology, they are denoted by $D_{A}^{(1)}(f), D_{A}^{(2)}\left(f_{1}, f_{2}\right)$, and $D_{A}^{(3)}\left(f_{1}, f_{2}, f_{3}\right)$, where now the subscript " $T=A$ " means the whole amplifier.

After performing the needed algebraic operations on equations of (19), we get

$$
\begin{gathered}
D_{A}^{(1)}(f)=\frac{X_{g o r}^{(1)}(f)}{X_{s}(f)}= \\
=\frac{D_{H D}^{(1)}(f)+g_{m 1}\left(D_{H 2}^{(1)}(f) D_{H 1}^{(1)}(f)-D_{H F}^{(1)}(f) D_{H D}^{(1)}(f)\right)}{1-g_{m 1} D_{H F}^{(1)}(f)-g_{o 1} D_{H 2}^{(1)}(f)}
\end{gathered}
$$

In [5], the linear transfer functions $D_{H D}^{(1)}, D_{H 2}^{(1)}, D_{H 1}^{(1)}$, and $D_{H F}^{(1)}$ are denoted by $H_{D}, H_{2}, H_{1}$, and $H_{F}$, respectively. Knowing this, one identifies easily the same expression as (22) in the paper by Miao and Zhang.

As it will be seen, the following linear transfer function

$$
\begin{gathered}
\tilde{D}_{A}^{(1)}(f)=\frac{X_{g m r}^{(1)}(f)}{X_{s}(f)}= \\
=\frac{D_{H 1}^{(1)}(f)+g_{o 1}\left(D_{H F}^{(1)}(f) D_{H D}^{(1)}(f)-D_{H 2}^{(1)}(f) D_{H 1}^{(1)}(f)\right)}{1-g_{m 1} D_{H F}^{(1)}(f)-g_{o 1} D_{H 2}^{(1)}(f)}
\end{gathered}
$$

which one obtains also from equations (19), will be very helpful in further calculations.

From (20), with help of (22) and (23), we get the following expression for the second-order nonlinear transfer function of the whole amplifier of Fig. 9

$$
\begin{gathered}
D_{A}^{(2)}\left(f_{1}, f_{2}\right)=\frac{X_{g o r}^{(2)}\left(f_{1}, f_{2}\right)}{X_{s}\left(f_{1}\right) X_{s}\left(f_{2}\right)}= \\
=\frac{D_{H 2}^{(1)}\left(f_{1}+f_{2}\right)}{1-g_{m 1} D_{H F}^{(1)}\left(f_{1}+f_{2}\right)-g_{o 1} D_{H 2}^{(1)}\left(f_{1}+f_{2}\right)} \times \\
\times\left[g_{m 2} \tilde{D}_{A}^{(1)}\left(f_{1}\right) \tilde{D}_{A}^{(1)}\left(f_{2}\right)+\right. \\
\left.+g_{o 2} D_{A}^{(1)}\left(f_{1}\right) D_{A}^{(1)}\left(f_{2}\right)\right]
\end{gathered}
$$

Moreover, we also obtain, as a byproduct, an expression for another second-order transfer function defined as $\tilde{D}_{A}^{(2)}\left(f_{1}, f_{2}\right)=X_{g m r}^{(2)}\left(f_{1}, f_{2}\right) /\left[X_{s}\left(f_{1}\right) X_{s}\left(f_{2}\right)\right]$. That is

$$
\begin{gathered}
\tilde{D}_{A}^{(2)}\left(f_{1}, f_{2}\right)=\frac{X_{g m r}^{(2)}\left(f_{1}, f_{2}\right)}{X_{s}\left(f_{1}\right) X_{s}\left(f_{2}\right)}= \\
=\frac{D_{H F}^{(1)}\left(f_{1}+f_{2}\right)}{1-g_{m 1} D_{H F}^{(1)}\left(f_{1}+f_{2}\right)-g_{o 1} D_{H 2}^{(1)}\left(f_{1}+f_{2}\right)} \times \\
\times\left[g_{m 2} \tilde{D}_{A}^{(1)}\left(f_{1}\right) \tilde{D}_{A}^{(1)}\left(f_{2}\right)+\right. \\
\left.+g_{o 2} D_{A}^{(1)}\left(f_{1}\right) D_{A}^{(1)}\left(f_{2}\right)\right]= \\
=\frac{D_{H F}^{(1)}\left(f_{1}+f_{2}\right)}{D_{H 2}^{(1)}\left(f_{1}+f_{2}\right)} D_{A}^{(2)}\left(f_{1}, f_{2}\right)
\end{gathered}
$$

$\tilde{D}_{A}^{(2)}\left(f_{1}, f_{2}\right)$ given by (25) will be helpful in calculations of the third-order nonlinear transfer function $D_{A}^{(3)}\left(f_{1}, f_{2}, f_{3}\right)$. 
Finally, by carrying out the needed algebraic operations on equations (21) and exploiting also (22), (23), (24), and (25), we arrive at

$$
\begin{gathered}
D_{A}^{(3)}\left(f_{1}, f_{2}, f_{3}\right)=\frac{X_{g o r}^{(3)}\left(f_{1}, f_{2}, f_{3}\right)}{X_{s}\left(f_{1}\right) X_{s}\left(f_{2}\right) X_{s}\left(f_{3}\right)}= \\
=\frac{D_{H 2}^{(1)}\left(f_{1}+f_{2}+f_{3}\right)}{1-g_{m 1} D_{H F}^{(1)}\left(f_{1}+f_{2}+f_{3}\right)-g_{o 1} D_{H 2}^{(1)}\left(f_{1}+f_{2}+f_{3}\right)} \times \\
\times\left[g _ { m 2 } \left(\tilde{D}_{A}^{(1)}\left(f_{1}\right) \tilde{D}_{A}^{(2)}\left(f_{2}, f_{3}\right)+\right.\right. \\
\left.\quad+\tilde{D}_{A}^{(2)}\left(f_{1}, f_{2}\right) \tilde{D}_{A}^{(1)}\left(f_{3}\right)\right)+ \\
+g_{m 3} \tilde{D}_{A}^{(1)}\left(f_{1}\right) \tilde{D}_{A}^{(1)}\left(f_{2}\right) \tilde{D}_{A}^{(1)}\left(f_{3}\right)+ \\
+g_{o 2}\left(D_{A}^{(1)}\left(f_{1}\right) D_{A}^{(2)}\left(f_{2}, f_{3}\right)+\right. \\
\left.\quad+D_{A}^{(2)}\left(f_{1}, f_{2}\right) D_{A}^{(1)}\left(f_{3}\right)\right)+ \\
\left.+g_{o 3} D_{A}^{(1)}\left(f_{1}\right) D_{A}^{(1)}\left(f_{2}\right) D_{A}^{(1)}\left(f_{3}\right)\right]
\end{gathered}
$$

Expression (26) provides us a formula for calculation of the third-order nonlinear transfer function of the whole amplifier structure shown in Fig. 9.

Note now that the expressions (22), (24), and (26) for the amplifier nonlinear transfer functions we derived in this section are more general than their harmonic distortion counterparts called nonlinear coefficients (of the corresponding orders), developed in [5]. The latter ones are useless in evaluations of such nonlinear distortion measures as, for example, the intermodulation distortion coefficients of the second- and third-order [8], [12], [18] or the cross-modulation index [10], [15], calculated in such situations in which the input signal consists of two or more sinusoids of different frequencies. Then, as shown in the literature cited above, more general schemes are to be used, which exploit the notion of nonlinear transfer functions derived from the Volterra series.

We complement the material of this section by showing how to get the nonlinear coefficients presented in [5] using our expressions found for the amplifier nonlinear transfer functions. To this end, note that to perform the above task we must proceed according to the rules explained, for example, in [8], [12], [18]. That is, having the input signal of the form

$$
x_{s}(t)=A_{o} \exp \left(j 2 \pi f_{o} t\right) \Leftrightarrow X_{s}(f)=A_{o} \delta\left(f-f_{o}\right)
$$

where $A_{o}$ and $f_{o}$ mean the amplitude and frequency of the complex harmonic signal $x_{s}(t)$, and $\delta$ stands for the Dirac impulse, we need only to substitute $f=f_{1}=f_{o}, f_{2}=f_{o}$, and $f_{3}=f_{o}$ in (22), (24), and (26). Then, we get successively

$$
\begin{gathered}
D_{A}^{(1)}\left(f_{o}\right)= \\
=\frac{D_{H D}^{(1)}\left(f_{o}\right)+g_{m 1}\left(D_{H 2}^{(1)}\left(f_{o}\right) D_{H 1}^{(1)}\left(f_{o}\right)-D_{H F}^{(1)}\left(f_{o}\right) D_{H D}^{(1)}\left(f_{o}\right)\right)}{1-g_{m 1} D_{H F}^{(1)}\left(f_{o}\right)-g_{o 1} D_{H 2}^{(1)}\left(f_{o}\right)} \\
D_{A}^{(2)}\left(f_{o}, f_{o}\right)= \\
=\frac{D_{H 2}^{(1)}\left(2 f_{o}\right)\left[g_{m 2}\left(\tilde{D}_{A}^{(1)}\left(f_{o}\right)\right)^{2}+g_{o 2}\left(D_{A}^{(1)}\left(f_{o}\right)\right)^{2}\right]}{1-g_{m 1} D_{H F}^{(1)}\left(2 f_{o}\right)-g_{o 1} D_{H 2}^{(1)}\left(2 f_{o}\right)}
\end{gathered}
$$

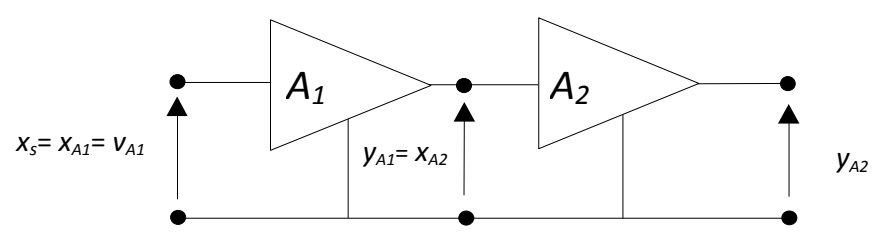

Fig. 10. Cascade connection of two amplifiers.

$$
\begin{gathered}
D_{A}^{(3)}\left(f_{o}, f_{o}, f_{o}\right)= \\
\frac{D_{H 2}^{(1)}\left(3 f_{o}\right)}{1-g_{m 1} D_{H F}^{(1)}\left(3 f_{o}\right)-g_{o 1} D_{H 2}^{(1)}\left(3 f_{o}\right)} \times \\
\times\left[2 g_{m 2} \tilde{D}_{A}^{(1)}\left(f_{o}\right) \tilde{D}_{A}^{(2)}\left(f_{o}, f_{o}\right)+\right. \\
+g_{m 3}\left(\tilde{D}_{A}^{(1)}\left(f_{o}\right)\right)^{3}+ \\
+2 g_{o 2} D_{A}^{(1)}\left(f_{o}\right) D_{A}^{(2)}\left(f_{o}, f_{o}\right)+ \\
\left.+g_{o 3}\left(D_{A}^{(1)}\left(f_{o}\right)\right)^{3}\right]
\end{gathered}
$$

The functions given by (28a), (28b), and (28c) are the nonlinear coefficients of the first-, second-, and third- order, respectively - in the sense defined in [5] - of the whole amplifier structure shown in Fig. 9.

\section{IN-NETWORK DESCRIPTION OF TWO-STAGE}

\section{Amplifier Being Component of ThreE-Stage One}

At the beginning of this section, we remark once again that the nonlinear Rosenstark's amplifier model developed is treated by us exactly in the same way as all of its composing elements listed in Fig. 8. Namely, it is modeled as a generalized 2-terminal circuit element. And as such, it possesses (see Section 3) two descriptions in the frequency domain: in-network and input-output ones. The Fourier transforms of the nonlinear impulse responses of the latter one, $D_{A}^{(1)}(f), D_{A}^{(2)}\left(f_{1}, f_{2}\right)$, and $D_{A}^{(3)}\left(f_{1}, f_{2}, f_{3}\right)$, have been in fact derived in the previous section (for definition of this model in the frequency domain, see equations (14c)). Further, it follows from the previous derivations that we obtain the in-network representation in the frequency domain for the nonlinear Rosenstark's amplifier model by substitution of $D_{T}^{(1)}(f)=D_{A}^{(1)}(f), D_{T}^{(2)}\left(f_{1}, f_{2}\right)=D_{A}^{(2)}\left(f_{1}, f_{2}\right)$, and $D_{T}^{(3)}\left(f_{1}, f_{2}, f_{3}\right)=D_{A}^{(3)}\left(f_{1}, f_{2}, f_{3}\right)$ in equations (13b)-(13d).

Consider now a cascade connection of two amplifiers $A_{1}$ and $A_{2}$ (denoted later also as $A 1$ and $A 2$, respectively, in subscripts of their nonlinear transfer functions and input/output variables). It is shown in Fig. 10.

Using the assumption that the amplifiers can be described by our nonlinear Rosenstark's amplifier model developed, we can redraw the schematic of Fig. 10 - applying the notion of equivalent generalized 2-terminal circuit element presented in Fig. 7 - in the form given by a scheme of Fig. 11 .

Note that to write now the equations describing the connection in Fig. 11 in the frequency domain, we need to use the 


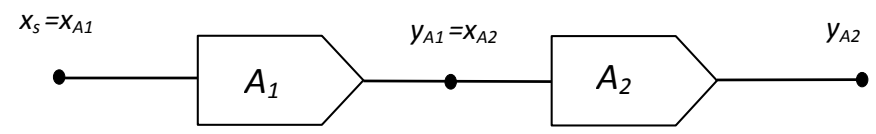

Fig. 11. Presentation of amplifier cascade connection with use of 2-node oriented graphs defined in Fig. 7.

input-output description (equations (14c)) for the amplifier $A_{1}$ and the in-network representation (equations (13b)-(13d)) for the amplifier $A_{2}$. Applying this, we get the following sets of equations

$$
\begin{gathered}
\left\{\begin{array}{c}
Y_{A 1}^{(1)}(f)=D_{A 1}^{(1)}(f) X_{s}(f) \\
X_{A 2 r}^{(1)}(f)=Y_{A 1}^{(1)}(f) \\
Y_{A 2 r}^{(1)}(f)=D_{A 2}^{(1)}(f) X_{A 2 r}^{(1)}(f)
\end{array}\right. \\
\left\{\begin{array}{c}
Y_{A 1}^{(2)}\left(f_{1}, f_{2}\right)=D_{A 1}^{(2)}\left(f_{1}, f_{2}\right) X_{s}^{(1)}\left(f_{1}\right) X_{s}^{(1)}\left(f_{1}\right) \\
X_{A 2 r}^{(2)}\left(f_{1}, f_{2}\right)=Y_{A 1}^{(2)}\left(f_{1}, f_{2}\right) \\
Y_{A 2 r}^{(2)}\left(f_{1}, f_{2}\right)=D_{A 2}^{(1)}\left(f_{1}+f_{2}\right) X_{A 2 r}^{(2)}\left(f_{1}, f_{2}\right)+ \\
+D_{A 2}^{(2)}\left(f_{1}, f_{2}\right) X_{A 2 r}^{(1)}\left(f_{1}\right) X_{A 2 r}^{(1)}\left(f_{2}\right)
\end{array}\right. \\
\left\{\begin{array}{c}
Y_{A 1}^{(3)}\left(f_{1}, f_{2}, f_{3}\right)=D_{A 1}^{(3)}\left(f_{1}, f_{2}, f_{3}\right) \times \\
\times X_{s}\left(f_{1}\right) X_{s}\left(f_{2}\right) X_{s}\left(f_{3}\right) \\
X_{A 2 r}^{(3)}\left(f_{1}, f_{2}, f_{3}\right)=Y_{A 1}^{(3)}\left(f_{1}, f_{2}, f_{3}\right) \\
Y_{A 2 r}^{(3)}\left(f_{1}, f_{2}, f_{3}\right)= \\
=D_{A 2}^{(1)}\left(f_{1}+f_{2}+f_{3}\right) X_{A 2 r}^{(3)}\left(f_{1}, f_{2}, f_{3}\right)+ \\
+D_{A 2}^{(2)}\left(f_{1}, f_{2}+f_{3}\right) X_{A 2 r}^{(1)}\left(f_{1}\right) X_{A 2 r}^{(2)}\left(f_{2}, f_{3}\right)+ \\
+D_{A 2}^{(2)}\left(f_{1}+f_{2}, f_{3}\right) X_{A 2 r}^{(2)}\left(f_{1}, f_{2}\right) X_{A 2 r}^{(1)}\left(f_{3}\right)+ \\
+D_{A 2}^{(3)}\left(f_{1}, f_{2}, f_{3}\right) X_{A 2 r}^{(1)}\left(f_{1}\right) \times \\
\times X_{A 2 r}^{(1)}\left(f_{2}\right) X_{A 2 r}^{(1)}\left(f_{3}\right)
\end{array}\right.
\end{gathered}
$$

In the next step, after performing the needed algebraic operations on equations of (27), we arrive at

$$
D_{C A}^{(1)}(f)=\frac{X_{A 2 r}^{(1)}(f)}{X_{s}(f)}=D_{A 2}^{(1)}(f) D_{A 1}^{(1)}(f)
$$

being the nonlinear transfer function of the first-order (the linear one) of the cascade of amplifiers $A_{1}$ and $A_{2}$ (denoted by the letters " $C A$ " in the subscript of $D_{C A}^{(1)}(f)$ ). And similarly, from (30) with application of (29) and from (31) with application of (29) and (30), we get, respectively

$$
\begin{gathered}
D_{C A}^{(2)}\left(f_{1}, f_{2}\right)=\frac{X_{A 2 r}^{(2)}\left(f_{1}, f_{2}\right)}{X_{s}\left(f_{1}\right) X_{s}\left(f_{2}\right)}= \\
D_{A 2}^{(1)}\left(f_{1}+f_{2}\right) D_{A 1}^{(2)}\left(f_{1}, f_{2}\right)+ \\
+D_{A 2}^{(2)}\left(f_{1}, f_{2}\right) D_{A 1}^{(1)}\left(f_{1}\right) D_{A 1}^{(1)}\left(f_{2}\right)
\end{gathered}
$$

and

$$
\begin{gathered}
D_{C A}^{(3)}\left(f_{1}, f_{2}, f_{3}\right)=\frac{X_{A 2 r}^{(3)}\left(f_{1}, f_{2}, f_{3}\right)}{X_{s}\left(f_{1}\right) X_{s}\left(f_{2}\right) X_{s}\left(f_{3}\right)}= \\
=D_{A 2}^{(1)}\left(f_{1}+f_{2}+f_{3}\right) D_{A 1}^{(3)}\left(f_{1}, f_{2}, f_{3}\right)+ \\
+D_{A 2}^{(2)}\left(f_{1}, f_{2}+f_{3}\right) D_{A 1}^{(1)}\left(f_{1}\right) D_{A 1}^{(2)}\left(f_{2}, f_{3}\right)+ \\
+D_{A 2}^{(2)}\left(f_{1}+f_{2}, f_{3}\right) D_{A 1}^{(2)}\left(f_{1}, f_{2}\right) D_{A 1}^{(1)}\left(f_{3}\right)+ \\
+D_{A 2}^{(3)}\left(f_{1}, f_{2}, f_{3}\right) D_{A 1}^{(1)}\left(f_{1}\right) D_{A 1}^{(1)}\left(f_{2}\right) D_{A 1}^{(1)}\left(f_{3}\right)
\end{gathered}
$$

Obviously, the functions $D_{C A}^{(2)}\left(f_{1}, f_{2}\right)$ given by (33) and $D_{C A}^{(3)}\left(f_{1}, f_{2}, f_{3}\right)$ given by (34) are the nonlinear transfer functions of the second- and third-order, accordingly, of the amplifier cascade.

At this point, it is worth noting that the formulas (32), (33), and (34) for calculation of the nonlinear transfer functions of an amplifier cascade have been derived or rediscovered many times in the literature, see, for example, articles [11], [22], [23] for general framework formulations using the Volterra series, and the recent ones [5], [24], [25] presentations in the particular context of harmonic distortion, harmonic balance method, and use of phasors.

The formulas (32), (33), and (34) are needed in study of the nonlinear distortion measures of two-stage amplifiers. And note that such studies with regard to the intermodulation distortion measure were performed by Narayanan [22] already many years ago, for amplifiers using bipolar transistor technology. Recently, a number of articles [3], [5], [24], [25] on similar evaluations of nonlinear properties, but regarding now the twostage CMOS amplifiers, has been published. In these recent papers, the second- and third-order harmonic distortion factors are analysed.

It is clear that the analysis of a three-stage amplifier built as a cascade of single amplifier blocks can be performed using the results presented above. That is by carrying out twice the operation of cascading. This can be done, for example, by cascading first $A_{1}$ and $A_{2}$ as shown in Fig. 11 to provide a twostage "post-amplifier", which is next cascaded according to the same rules with a single-stage "pre-amplifier" - altogether resulting in the three-stage amplification block. And it is evident that in such the procedure, we need to have the in-network description of the cascade of amplifiers $A_{1}$ and $A_{2}$ (which, as we know, differs from its input-output representation derived just before). However, its development, though being a little bit cumbersome, is straightforward. Simply then, we use the generic procedure for this case defined by equations (13b)(13d) together with the expressions for the nonlinear transfer functions given by (32), (33), and (34). This results in

$$
\begin{gathered}
Y_{C A r}^{(1)}(f)=D_{A 2}^{(1)}(f) D_{A 1}^{(1)}(f) X_{C A r}^{(1)}(f) \\
Y_{C A r}^{(2)}\left(f_{1}, f_{2}\right)= \\
=D_{A 2}^{(1)}\left(f_{1}+f_{2}\right) D_{A 1}^{(1)}\left(f_{1}+f_{2}\right) X_{C A r}^{(2)}\left(f_{1}, f_{2}\right)+ \\
+\left[D_{A 2}^{(1)}\left(f_{1}+f_{2}\right) D_{A 1}^{(2)}\left(f_{1}, f_{2}\right)+\right. \\
\left.+D_{A 2}^{(2)}\left(f_{1}, f_{2}\right) D_{A 1}^{(1)}\left(f_{1}\right) D_{A 1}^{(1)}\left(f_{2}\right)\right] \times \\
\times X_{C A r}^{(1)}\left(f_{1}\right) X_{C A r}^{(1)}\left(f_{2}\right) \\
\quad Y_{C A r}^{(3)}\left(f_{1}, f_{2}, f_{3}\right)=D_{A 2}^{(1)}\left(f_{1}+f_{2}+f_{3}\right) \times \\
\times D_{A 1}^{(1)}\left(f_{1}+f_{2}+f_{3}\right) X_{C A r}^{(3)}\left(f_{1}, f_{2}, f_{3}\right)+ \\
+\left[D_{A 2}^{(1)}\left(f_{1}+f_{2}+f_{3}\right) D_{A 1}^{(2)}\left(f_{1}, f_{2}+f_{3}\right)+\right. \\
\left.+D_{A 2}^{(2)}\left(f_{1}, f_{2}+f_{3}\right) D_{A 1}^{(1)}\left(f_{1}\right) D_{A 1}^{(1)}\left(f_{2}+f_{3}\right)\right] \times \\
X_{C A r}^{(1)}\left(f_{1}\right) X_{C A r}^{(2)}\left(f_{2}, f_{3}\right)+\left[D_{A 2}^{(1)}\left(f_{1}+f_{2}+f_{3}\right) \times\right. \\
\times D_{A 1}^{(2)}\left(f_{1}+f_{2}, f_{3}\right)+D_{A 2}^{(2)}\left(f_{1}+f_{2}, f_{3}\right) \times \\
\left.\times D_{A 1}^{(1)}\left(f_{1}+f_{2}\right) D_{A 1}^{(1)}\left(f_{3}\right)\right] \times
\end{gathered}
$$




$$
\begin{gathered}
\times X_{C A r}^{(2)}\left(f_{1}, f_{2}\right) X_{C A r}^{(1)}\left(f_{3}\right)+ \\
+\left[D_{A 2}^{(1)}\left(f_{1}+f_{2}+f_{3}\right) D_{A 1}^{(3)}\left(f_{1}, f_{2}, f_{3}\right)+\right. \\
+D_{A 2}^{(2)}\left(f_{1}, f_{2}+f_{3}\right) D_{A 1}^{(1)}\left(f_{1}\right) D_{A 1}^{(2)}\left(f_{2}, f_{3}\right)+ \\
+D_{A 2}^{(2)}\left(f_{1}+f_{2}, f_{3}\right) D_{A 1}^{(2)}\left(f_{1}, f_{2}\right) D_{A 1}^{(1)}\left(f_{3}\right)+ \\
\quad+D_{A 2}^{(3)}\left(f_{1}, f_{2}, f_{3}\right) \times \\
\left.\times D_{A 1}^{(1)}\left(f_{1}\right) D_{A 1}^{(1)}\left(f_{2}\right) D_{A 1}^{(1)}\left(f_{3}\right)\right] \times \\
\times X_{C A r}^{(1)}\left(f_{1}\right) X_{C A r}^{(1)}\left(f_{2}\right) X_{C A r}^{(1)}\left(f_{3}\right)
\end{gathered}
$$

with, obviously, $X_{C A r}^{(i)}=X_{C A 1}^{(i)}, Y_{C A r}^{(i)}=Y_{A 2 r}^{(i)}, i=1,2,3$, and $X_{C A r}^{(1)} \neq X_{s}$.

Finally in this section, we draw the reader's attention to the fact that the in-network description of the cascade of two amplifiers, given by equations (35), (36), and (37), has been developed here for the first time in the literature.

\section{Remarks on Some Questionable Notions}

In the paper [5], Miao and Zhang use some specific notions and concepts, which are highly questionable as, for example, the following ones:

1) nonlinear signals,

2) intermodulation nonlinearity,

3) second-order intermodulation nonlinearity,

4) nonlinear gain function at fundamental frequency,

5) identification of transfer function poles and zeros lying on the real axis of the complex plane with the related frequencies, being positive real numbers.

Let us explain here in more detail their incorrectness and recall the proper names and their usage.

With regard to nonlinear signals: Note that signals are neither linear nor nonlinear. After performing any nonlinear processing on a signal, it remains still a signal, assuming only another form. Linear or nonlinear can be systems, not signals.

With regard to intermodulation nonlinearity: Such notions as, for example, intermodulation, harmonic or cross-modulation nonlinearities do not make sense. We explain this point on an example of a circuit element realizing a quadratic nonlinearity $y_{T}(t)=a \cdot x_{T}^{2}(t)$, with the coefficient " $a$ " being a real number. Applying input signal $x_{T}(t)=A_{o} \exp \left(j 2 \pi f_{o} t\right.$ ) (as described by (27)) to it, we get the output signal in the form: $y_{T}(t)=a \cdot A_{o}^{2} \exp \left(j 2 \pi\left(2 f_{o}\right) t\right)$. Observe that this resulting signal is a pure harmonic one at the product frequency $2 f_{o}$. Next, using another input signal $x_{T}(t)=A_{o 1} \exp \left(j 2 \pi f_{o 1} t\right)+$ $A_{o 2} \exp \left(j 2 \pi f_{o 2} t\right)$, being a sum of the two previous signals at different frequencies $f_{o 1}$ and $f_{o 2}$, in the above quadratic nonlinearity, we obtain $y_{T}(t)=a \cdot A_{o 1}^{2} \exp \left(j 2 \pi\left(2 f_{o 1}\right) t\right)+$ $a \cdot A_{o 2}^{2} \exp \left(j 2 \pi\left(2 f_{o 2}\right) t\right)+2 a \cdot A_{o 1} A_{o 2} \exp \left(j 2 \pi\left(f_{o 1}+f_{o 2}\right) t\right)$ in this case. And note that the output signal consists now of two harmonics of frequencies $2 f_{o 1}$ and $2 f_{o 2}$, respectively, and of the intermodulation product at frequency $f_{o 1}+f_{o 2}$.

Comparison of the above two results leads to the conclusion that the quadratic nonlinearity considered can produce both the harmonic as well as the intermodulation products (depending upon the form of the input signal). So, therefore, it is neither harmonic nor intermodulation nonlinearity. It is simply a quadratic nonlinearity - and this is its proper name.

With regard to second-order intermodulation nonlinearity:
This notion is also flawed. To see this, recall the example of quadratic nonlinearity from the previous point with the input signal $x_{T}(t)=A_{o 1} \exp \left(j 2 \pi f_{o 1} t\right)+A_{o 2} \exp \left(j 2 \pi f_{o 2} t\right)$ applied to it. This results, as shown above, in $y_{T}(t)=$ $a \cdot A_{o 1}^{2} \exp \left(j 2 \pi\left(2 f_{o 1}\right) t\right)+a \cdot A_{o 2}^{2} \exp \left(j 2 \pi\left(2 f_{o 2}\right) t\right)+2 a$. $A_{o 1} A_{o 2} \exp \left(j 2 \pi\left(f_{o 1}+f_{o 2}\right) t\right)$, that is in two second-order harmonic distortion components $a \cdot A_{o 1}^{2} \exp \left(j 2 \pi\left(2 f_{o 1}\right) t\right)$ and $a \cdot A_{o 2}^{2} \exp \left(j 2 \pi\left(2 f_{o 2}\right) t\right)$, and the second-order intermodulation distortion one $2 a \cdot A_{o 1} A_{o 2} \exp \left(j 2 \pi\left(f_{o 1}+f_{o 2}\right) t\right)$. Therefore, the above nonlinearity cannot be solely named a secondorder intermodulation nonlinearity.

With regard to nonlinear gain function at fundamental frequency: The above notion is conceptually false because there exists no such a single nonlinear gain function, which enables calculation of the gains for the corresponding harmonics and the frequencies of intermodulation products, and for the fundamental frequency. For calculation of the gain at the fundamental frequency, one has to use a separate function called in the literature the describing function [10], [26]. Further, for evaluation of the gain of the second-order harmonic, another function must be used - and so on - for other harmonics and frequencies of intermodulation products.

With regard to making identity between the transfer function poles and zeros, and the related frequencies: One calculates single poles and zeros of the transfer functions by equating to zero the polynomials of the form $\left(s+a_{p}\right)$ and $\left(s+a_{z}\right)$, with $a_{p}$ and $a_{z}$ being real positive numbers. Hence, the pole and zero positions are given by $s_{p}=-a_{p}$ and $s_{z}=-a_{z}$, which lie on the real axis of the complex s-plane in its left-hand side. These positions are identified in [5] with the related angular frequencies, which are obviously positive numbers. Correctly, the related pole and zero frequencies should be written as $\omega_{p}=\left|s_{p}\right|=a_{p}$ and $\omega_{z}=\left|s_{z}\right|=a_{z}$, respectively.

Furthermore, it should be stressed that the use of the notion of poles and zeros of the nonlinear coefficients and harmonic distortion factors of the second- and third-order, as done in [5], cannot be extended to the other areas as, for instance, to stability issues (at least in the form presented in [5]).

\section{CONCLUSION}

In this paper, a thorough and mathematically rigorous extension of the linear Rosenstark amplifier model to a weakly nonlinear one has been presented. It has been also demonstrated that our model exploiting the Volterra series and the notion of generalized two-terminal nonlinear circuit elements is more general than the one using phasors [5], and that it assumes two forms, named here in-network and input-output type descriptions of an amplifier. In the paper, the relations describing these forms have been derived in the time as well as in the frequency domain. Moreover, it has been shown how to use them in calculations of any nonlinear distortion measure. In contrast to this, note that the method of phasors enables calculations of harmonic distortion only. Furthermore, note likewise that the descriptions developed enable simple calculation of the nonlinear transfer functions of any amplifier topology as, for example, cascade and feedback structures and their combinations occurring in single-, two-, and three-stage 
amplifiers. Two examples presented illustrate details of such calculations. Finally, we draw the reader's attention to the fact that many outcomes of this paper systematize, in some aspects, the results achieved recently with the use of the symbolic analysis applied to harmonic distortion analyses of CMOS amplifiers [3], [5], [13], [24], [25].

\section{REFERENCES}

[1] S. Rosenstark, "A simplified method of feedback amplifier analysis," IEEE Transactions on Education, vol. E-17, pp. 192-198, November 1974.

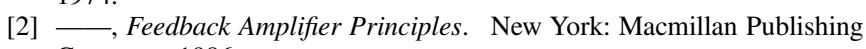
Company, 1986.

[3] G. Palumbo and S. Pennisi, "High-frequency harmonic distortion in feedback amplifiers: analysis and applications," IEEE Transactions on Circuits and Systems-I: Fundamental Theory and Applications, vol. 50, pp. 328-340, March 2003.

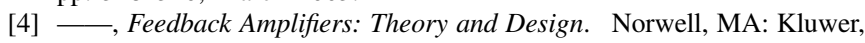
2002.

[5] Y. Miao and Y. Zhang, "Distortion modeling of feedback two-stage amplifier compensated with Miller capacitor and nulling resistor,' IEEE Transactions on Circuits and Systems-I: Regular Papers, vol. 59, pp. 93-105, January 2012.

[6] L. O. Chua, "Device modeling via basic nonlinear circuit elements," IEEE Transactions on Circuits Systems, vol. CAS-27, pp. 1014-1044, November 1980

[7] A. Borys, "On in-network and other types of amplifier descriptions for nonlinear distortion analysis," International Journal of Electronics and Telecommunications, vol. 56, no. 2, pp. 177-184, 2010.

[8] J. J. Bussgang, L. Ehrman, and J. W. Graham, "Analysis of nonlinear systems with multiple inputs," Proceedings of the IEEE, vol. 62, pp. 1088-1119, 1974.

[9] E. Bedrosian and S. O. Rice, "The output properties of Volterra systems (nonlinear systems with memory) driven by harmonic and Gaussian inputs," Proceedings of the IEEE, vol. 59, pp. 1688-1707, December 1971.

[10] L. O. Chua and C. Ng, "Frequency domain analysis of nonlinear systems: general theory," IEE Journal of Electronic Circuits and Systems, vol. 3, pp. 165-185, 1979.

[11] — , "Frequency domain analysis of nonlinear systems: formulation of transfer functions," IEE Journal of Electronic Circuits and Systems, vol. 3, pp. 257-269, 1979.

[12] A. Borys, "A simplified analysis of nonlinear distortion in analog electronic circuits using the Volterra-Wiener series," Scientia Electrica, vol. 30, no. 3, pp. 78-103, 1984.
[13] S. O. Cannizzaro, G. Palumbo, and S. Pennisi, "Effects of nonlinear feedback in the frequency domain," IEEE Transactions on Circuits and Systems-I: Fundamental Theory and Applications, vol. 53, pp. 225-234, February 2006.

[14] A. Borys, "On correctness of the basics of Palumbo and Pennisi's means of harmonic distortion calculation in analog integrated circuits," Zeszyty Naukowe Wyszej Szkoy Informatyki $w$ Łodzi: Teoria $i$ zastosowania informatyki, vol. 8, no. 2, pp. 5-16, 2009.

[15] R. G. Meyer, M. J. Shensa, and R. Eschenbach, "Crossmodulation and intermodulation in amplifiers at high frequencies," IEEE Journal of Solid-State Circuits, vol. SC-7, pp. 16-23, February 1972.

[16] A. Borys, "Exploiting admittance formalism in the nonlinear analysis," Zeszyty Naukowe Wyszej Szkoy Informatyki w L odzi: Teoria i zastosowania informatyki, vol. 8, no. 2, pp. 17-39, 2009.

[17] G. Palumbo, M. Pennisi, and S. Pennisi, "Miller theorem for weakly nonlinear feedback circuits and application to CE amplifier," IEEE Transactions on Circuits and Systems-II: Express Briefs, vol. 55, pp. 991-995, October 2008.

[18] S. Narayanan, "Application of Volterra series to intermodulation distortion analysis of transistor feedback amplifiers," IEEE Transactions on Circuit Theory, vol. CT-17, pp. 518-527, November 1970.

[19] P. Wambacq, G. Gielen, P. Kinget, and W. Sansen, "High-frequency distortion analysis of analog integrated circuits," IEEE Transactions on Circuits and Systems II: Analog and Digital Signal Processing, vol. 46, no. 3, pp. 335-344, March 1999.

[20] B. Hernes and W. Sansen, "Distortion in single-, two- and three-stage amplifiers," IEEE Transactions on Circuits and Systems I: Regular Papers, vol. 52, no. 5, pp. 846-856, May 2005.

[21] M. Fliess, M. Lamnabhi, and F. Lamnabhi-Lagarrigu, "An algebraic approach to nonlinear functional expansions," IEEE Transactions on Circuits and Systems, vol. 30, no. 8, pp. 554-570, 1983.

[22] S. Narayanan, "Intermodulation distortion of cascaded transistors," IEEE Journal of Solid-State Circuits, vol. SC-4, no. 3, pp. 97-106, June 1969.

[23] Y. L. Kuo, "Frequency-domain analysis of weakly nonlinear networks. "Canned" Volterra analysis, part 1," IEEE Circuits and Systems Magazine, vol. 11, no. 4, pp. 2-8, August 1977.

[24] S. O. Cannizzaro, G. Palumbo, and S. Pennisi, "Distortion analysis of Miller-compensated three-stage amplifiers," IEEE Transactions on Circuits and Systems I: Regular Papers, vol. 53, no. 5, pp. 961-976, May 2006.

[25] — , "An approach to model high-frequency distortion in negativefeedback amplifiers," International Journal of Circuit Theory and Applications, vol. 36, pp. 3-18, 2008.

[26] A. Gelb and W. E. Vander Velde, Multiple-Input Describing Functions and Nonlinear System Design. New York: McGraw-Hill, 1968. 